\title{
Automated Visual Defect Detection for Flat Steel Surface: A Survey
}

\author{
Qiwu Luo, Member, IEEE, Xiaoxin Fang, Li Liu, Chunhua Yang, Member, IEEE, and Yichuang Sun, \\ Senior Member, IEEE
}

\begin{abstract}
Automated computer-vision-based defect detection has received much attention with the increasing surface quality assurance demands for the industrial manufacturing of flat steels. This paper attempts to present a comprehensive survey on surface defect detection technologies by reviewing about 120 publications over the last two decades for three typical flat steel products of con-casting slabs, hot- and cold-rolled steel strips. According to the nature of algorithms as well as image features, the existing methodologies are categorized into four groups: Statistical, spectral, model-based and machine learning. These literatures are summarized in this review to enable easy referral to suitable methods for diverse application scenarios in steel mills. Realization recommendations and future research trends are also addressed at an abstract level.
\end{abstract}

Index Terms-Automated visual inspection (AVI), automated optical inspection (AOI), surface defect detection, flat steel, survey.

\section{INTRODUCTION}

A S A DOMINANT steel product, flat steel occupies more than $65 \%$ of all the products in the iron and steel industry, which is the vital fundamental material for the related planar industries, including without limitation, architecture, aerospace, machinery, automobile, and so on. Any quality problems suffering on flat steel would lead to huge economic and reputation losses to steel manufacturers. For thin and wide flat steel, surface defects are the greatest threat to the product quality. Even for occasional internal defects, morphological changes will arise on the surface with large probability. Automated visual inspection (AVI) instrument targeting on surface quality emerges as a standard configuration for flat steel mills to improve product quality and promote production efficiency.

A general AVI instrument provides two main functions of

This work was supported in part by the National Natural Science Foundation of China under Grant 51704089 and Grant 61973323 , in part by the Anhui Provincial Natural Science Foundation of China under Grant 1808085QF190.

Q. Luo and C. Yang are with the School of Automation, Central South University, Changsha 410083, China. (Corresponding Author, Chunhua Yang, Email:ychh@csu.edu.cn).

$\mathrm{X}$. Fang is with the School of Electrical and Automation Engineering, Hefei University of Technology, Hefei 230009, China.

L. Liu is with Center for Machine Vision and Signal Analysis, University of Oulu, Oulu 90014, Finland. She is also with the College of System Engineering, National University of Defense Technology, Changsha 410073, China.

Y. Sun is with the School of Engineering and Computer Science, University of Hertfordshire, Hatfield AL10 9AB, U.K.

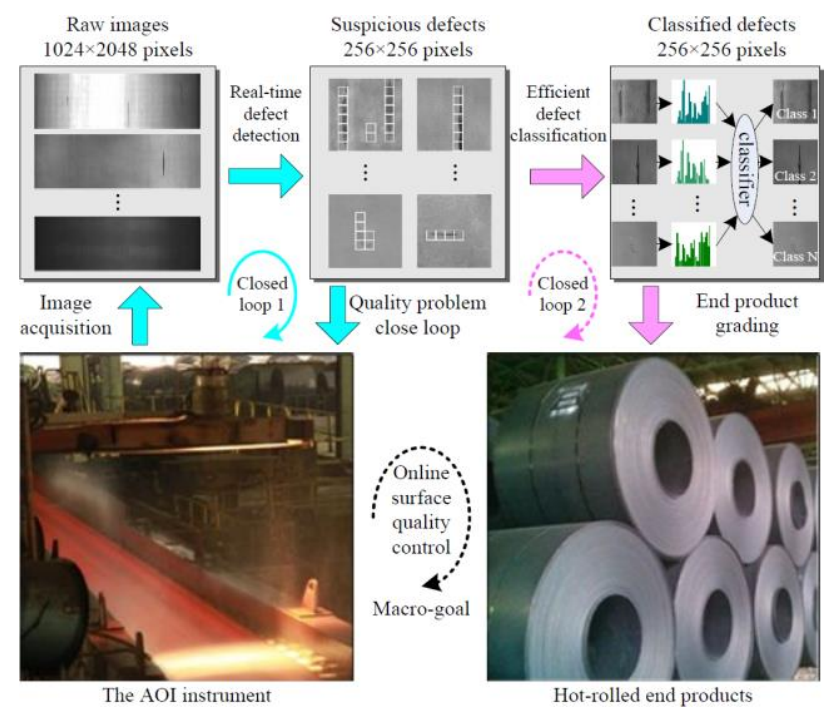

Fig. 1. The contribution of defect detection in a typical AVI instrument.

defect detection and classification [1-4]. The former detection process recognizes defective regions from normal background without identifying what types of defects they are. This step is the foundation of the "quality problem close loop", earlier defect detection allows less economic losses. The latter process is dedicated to identify and label detected defects to support finishing product grading. In this context, the flat steel covers three categories of con-casting slabs, hot- and cold-rolled steel strips, where slabs are rolled into hot strips and then to cold strips. Taking hot strip as an example, Fig. 1 briefly gives the flow chart of AVI processes. In general, defect detection is required to be in strict real-time while defect classification can be handled in quasi real-time. The total performance of AVI system is mainly limited by the accuracy, time-efficiency and robustness of the arithmetical methods in the defect detection process which is the very focus of this paper.

However, on-site surface defect detection in real-world steel mills is seriously challenging: 1) Unsatisfactory imaging environments. Continuous casting and rolling production lines involve multiple sufferings of high temperature, dense mist, heavy cooling water drops [5], uneven illumination, stochastic noises [1,2], and aperiodic vibration [6]. The undesirable image quality requires preeminent detection algorithms to resist large intra-class variation and minor inter-class distance [1-4]. 2) Eternally continuous image streams. The online dual-surface quality measurement for average flat steel mills requires the surface AVI instrument to continuously process about 2.56 Gbps of image flows [5] to identify defective regions, which 
force the detection algorithms to achieve excellent balance between accuracy, computational complexity and reliability.

Over the years, industry and academia devote themselves to address the aforementioned challenges from hardware upgrade to algorithmic optimization. The hardware architecture based on either server expansion [7-9] or ASIC acceleration [5] has been opened in some recent reports. Furthermore, it is not easy to see dramatic hardware breakthroughs within relatively short time due to the limitation of Moore's law [10]. This review thus focuses on the latest theoretical and algorithmic advances of automated visual defect detection over the past two decades to enable easy referral to suitable methods for diverse application scenarios in steel mills. Especially the literatures over the last five years accounted for nearly $50 \%$.

The structure of this context is as follows. After the introduction in Section I, some relevant prior survey papers are briefly reviewed in Section II. Typical defect morphologies on flat steel surfaces are illustrated vividly in Section III. The four categories of defect detection approaches are presented in Section IV in detail. This paper is ended in Section V with the conclusion and comments on the realization recommendations and future research trends.

\section{PRIOR Literature REVIEW}

A number of AVI surveys (such as [11-13]) with a wide coverage of inspection problems can be available successively. Recently published surveys gradually pay more attention to specific planar materials like fabric [8] and semiconductor [14]. Notably, a brief but rare AVI review covering defect detection and classification techniques for steel products was reported [9], where nearly all types of steel products (slab, billet, plate, hot strip, cold strip, rod/bar) are involved at an overview level. It is widely recognized that AVI techniques are more suitable to inspect surface defects on sheet materials than on wire rod/bar with minor diameter or even special-shaped structures [15]. To further narrow the scope of [9], that is, concentrate on the vital defect detection process on only flat steel products, this paper attempts to present a first Transactions survey on this focused topic, so as to support the AVI applications for the relevant industrial manufacturing.

\section{DEFECT MORPHOLOGIES ON FLAT STEEL SURFACE}

Various defects on flat steel surface are generally caused by mechanical or metallurgical imperfection during the industrial manufacturing. To save paper space, we only take some surface defect image samples for hot-rolled steel strips and con-casting slabs by using the AVI instrument designed in [5] for illustration. Fig. 2(a) lists four raw defective images ( $4096 \times 1024$ pixel) acquired by the equipped line-scan camera. And Fig. 2(b) presents eighteen typical defect samples with $256 \times 256$ pixel obtained from raw images after defect detection process. These are roller marks, longitudinal scratches, horizontal scratches, inclusions, scarring, holes, waves, pitting, air bubbles, peeling, water droplets, convex bags, reticulations, star cracks, foreign bodies, heavy leather, wrinkles and longitudinal cracks, respectively. Finally, in Fig. 2(c), some longitudinal crack image samples of con-casting slabs are presented $(512 \times 512$ pixel), and this defect type is with high probability of occurrence on continuous casting line, which has great threat to the quality of downstream products. Besides the diversity and complexity of these defects, nearly all the challenges mentioned in Sec. I can be encountered in these image samples. For example, some pseudo defects of water droplets and mill scales are pretty commonly distributing on the surfaces of hot-rolled strips and casting slabs, which would trigger false detection. Another example, the image intensity is fairly inhomogeneous and varies actively.

\section{TAXonomy OF Defect Detection Methods}

This section presents a review on the prior techniques and models for defect detection of flat steel surfaces. In general, researchers categorize previously proposed methods into different groups based on the distinct features, while the taxonomy also varies from person to person. Timm et al. [16] broadly separated texture defect detection approaches into local and global groups. According to different technique roadmaps, defect detection methods are summarized as classification-, local-abnormalities-, and template-matching-based methods in [17]. Youkachen et al. [18] classified defect detection methods into probabilistic, statistical, proximity-based, deviation-based and network-based models. At the microscopic level, the flat steel surface inspection problem is essentially a texture analysis problem [8]. Normally, texture analysis problem can be solved by statistical-, spectral- and model-based methods. Notably, machine learning enjoys its popularity in computer vision in recent years, especially in texture analysis. Thus, as shown in Fig. 3, this paper classifies defect detection methods for flat steel surfaces into four categories: conventional statistical, spectral, model-based and emerging machine learning.

\section{A. Statistical}

Statistical approaches are frequently used to detect defects of flat steel surface by evaluating the regular and periodic distribution of pixel intensities. Eight representative statistical methods are briefly introduced as follows.

\section{1) Thresholding}

Thresholding methods are usually used to separate the defective regions on flat steel surfaces, which have been widely applied in online AVI systems [19, 20]. The traditional thresholding methods identify defects by comparing the value of image pixels to a fix number and turn the test image into a simple binary frame, which is sensitive to random noises and non-uniform illuminations. Djukic et al. [21] first estimated the probability distribution of pixel intensities from some defect-free hot-rolled steel images, which was considered as a basis for adaptively determining threshold. The dynamical thresholding procedure can then discriminately separate true defects from random noise. Further, Nand et al. [22] calculated the local entropy of defective and defect-free images respectively and extracted defective region of image by using background subtraction method by comparing their entropy, it is reported to perform better on detecting defective blocks of 

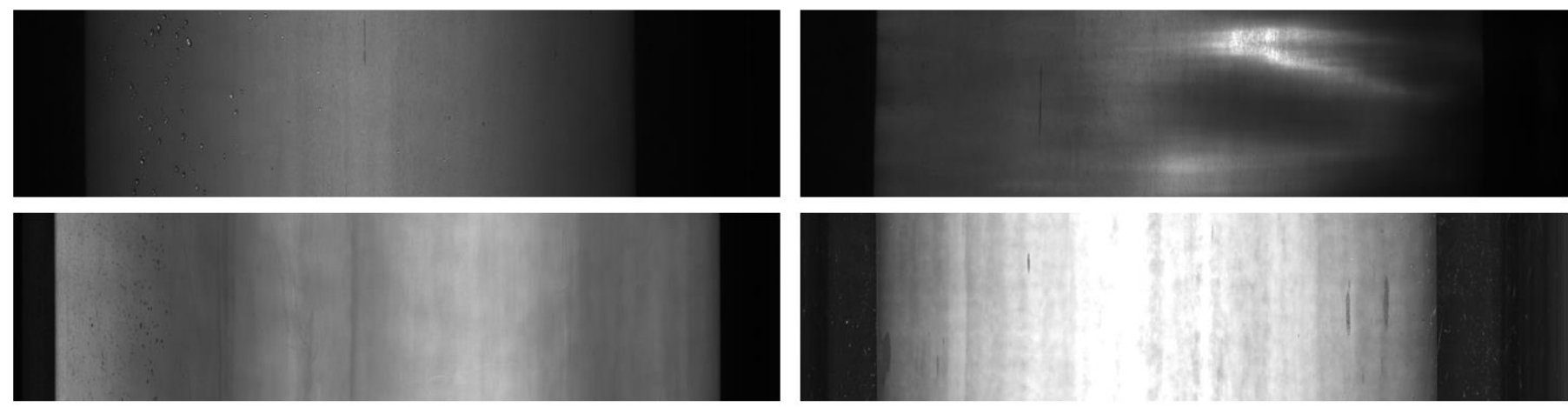

(a)
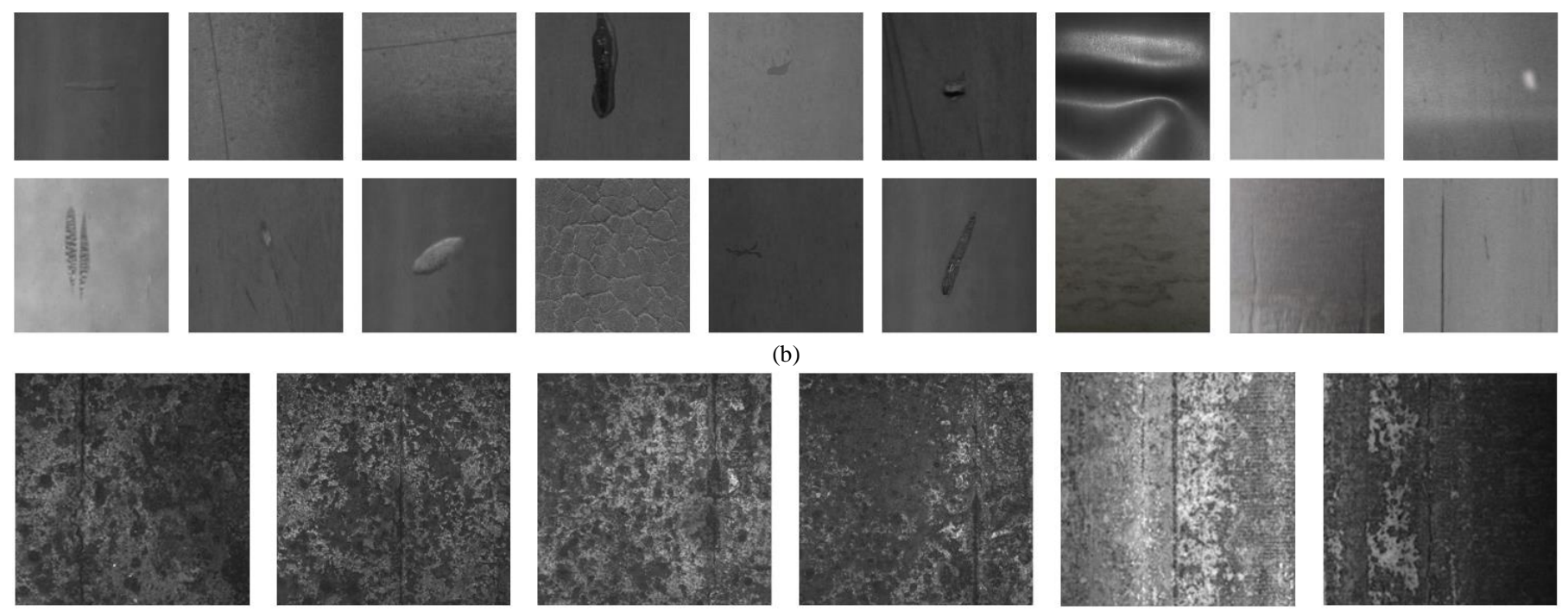

(b)
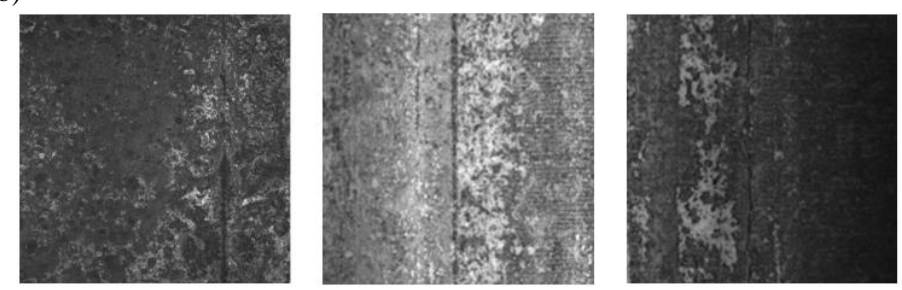

(c)

Fig. 2. Typical defect image samples. For hot-rolled steel strips: (a) are typical defective raw images of steel surface $(4096 \times 1024$ pixel) acquired by line-scan camera and (b) are a series of typical defect samples with $256 \times 256$ pixel. For con-casting slabs: (c) are typical longitudinal cracks acquired by area-scan camera.

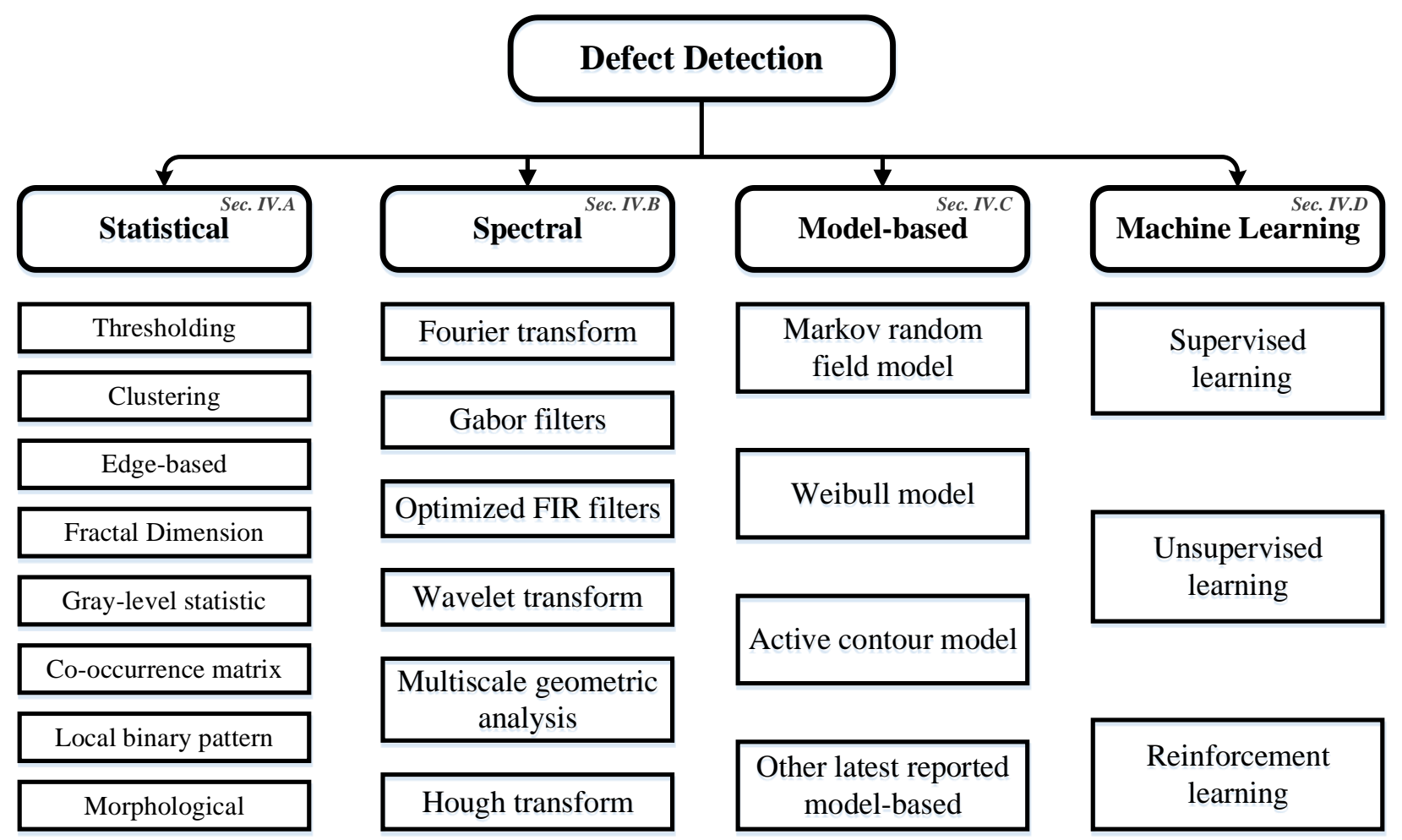

Fig. 3. The overall structure of detection method taxonomy. 
low-quality steel surface than former dynamical thresholding method. To obtain a better global detection performance, Neogi et al. [23] proposed a global adaptive percentile thresholding scheme based on gradient images. It can selectively segment defective region and effectively preserve the defect edges regardless of the size of defects. In order to further accomplish the task of defect detection, it is promising to obtain the optimal thresholds or design smarter dynamic thresholding mechanism.

\section{2) Clustering}

Based on the similarity among image pixels, clustering method is specialized in mining information implicitly existing in texture images, then defect detection can be achieved by the multiple-class defect classification. Real-time and anti-noise capability are always the basic requirements of industrial defect detection, Bulnes et al. [24] detected the defects occurring periodically by clustering the characteristics (i.e., position, type) of each defect. This method can timely detect periodical defects even in noisy environment. However, some interferences like stochastic industrial liquids increase the detection difficulty. Zhao et al. [25] proposed a two-level labeling technique to solve the above problem based on superpixels. The pixels are clustered into superpixels and then superpixels are clustered into subregions, the superpixel boundaries are updated iteratively until pixels with similar visual senses are clustered into one superpixel, subregions after many rounds of growth will converge towards defects. This method achieved an average correct detection rate of $91 \%$ when applying on cold strips. Further, Wang et al. [26] proposed an entity sparsity pursuit (ESP) method to detect surface defects. Defect image can be segmented into several superpixels to realize entity sparsity pursuit of defects, while defects do not satisfy the sparsity assumption in pixel level. The ESP method is insensitive to noise and computationally efficient. For the nature of clustering, it is more suitable for defect classification than defect detection.

\section{3) Edge-based}

The purpose of edge detection is to identify points with obvious brightness changes in digital images. Researchers often use local image differentiation technique to obtain edge detection operator, the commonly used edge detection templates for flat steel surface are Kirsch, Sobel and Canny operator. It is investigated that Sobel is specialized in weighing the influence of pixel position to reduce the ambiguity of edge, but it is sensitive to uneven illumination on flat steel surface, which easily leads to false edge detection. In order to avoid the false detection, Borselli et al. [27] modified Sobel operator by applying thresholding to convert the grayscale image to binary matrix. Further, Shi et al. in [28] developed eight directional templates to obtain more comprehensive edge information than the original Sobel operator which only has horizontal and vertical directions. Fig. 4 illustrates the technique details of these two Sobel operators including template topology, detect performance, etc. The easily trigged false edge detection was well suppressed by the eight-directional Sobel operator. With

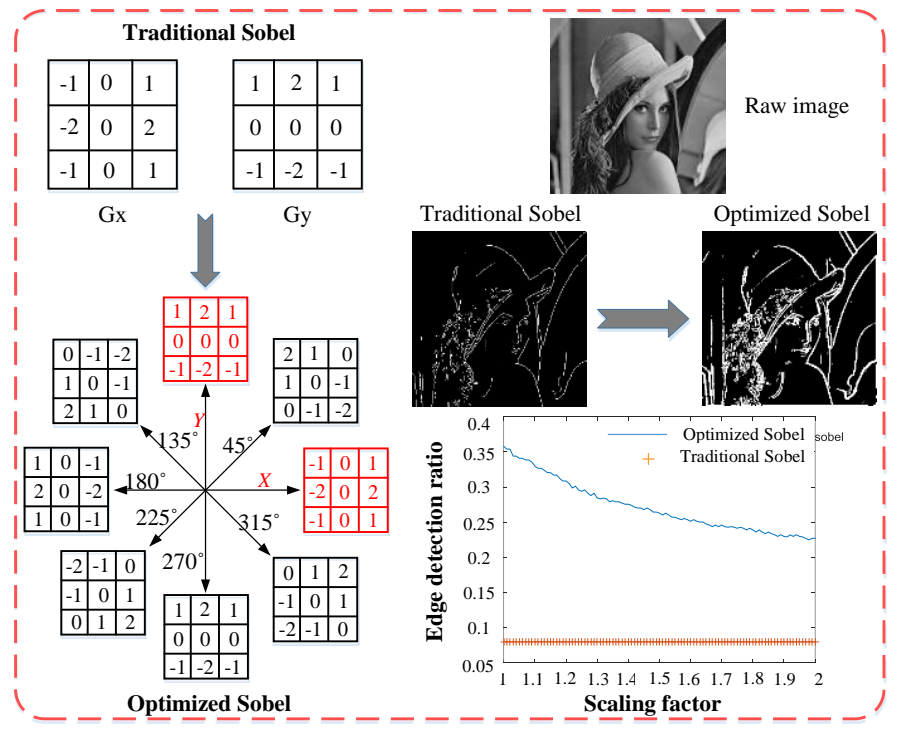

Fig. 4. Comparison of the traditional and the optimized Sobel operator.

the weighted factor and multiple templates, Kirsch is more noise-robust for tiny defect detection among flat steel images especially suffered with uneven illumination. While the eight directional templates bring large computation amounts to Kirsch. Bo et al. [29] simplified the original Kirsch operator by choosing some partial templates on the premise of little influence on edge extraction. Compared with the first-order Kirsch and Sobel operator, Canny possesses better signal-to-noise ratio and detection accuracy due to its second-order feature. However, it suffers with low adaptive ability and sometimes is easy to blur the noise-free region. Hence, it is not a wise choice to directly apply existing edge detection operator on steel surface defect inspection until appropriate algorithm is imported to enhance its edge detail retaining ability. Furthermore, many edge detection operators have not been used to detect surface defects of flat steel, such as Prewitt, Laplacian and Log. Specifically, Prewitt has been used for object enhancement and extraction. Laplacian sharpening template and Log operators have been reported performing well in determining edge position. So, it is highly recommended to explore other edge detection operators on the task of steel surface inspection in the near future.

\section{4) Fractal Dimension}

Fractal Dimension (FD) has the desirable self-similarity which means the overall information can be expressed by partial features. It is reported that statistical gray value of defect images practically possesses some features of FD, especially in self-similarity. Zhiznyakov et al. [30] employed fractal features of digital images to detect defects of flat steel surfaces by characterizing the internal distribution of self-similarity and the image segments with the highest similarity. The experimental results are basically consistent with inspected data from a non-destructive testing inspector. Similarly, multifractal dimension is utilized by Yazdchi et.al [31] to detach and specify the defective region for five typical defects of steel surfaces. It should be pointed out that the application of FD has some limitations because it is only suitable for self-similar defect image detection. 
TABLE I

List of Some of Typical Statistical Methods of Defect Detection

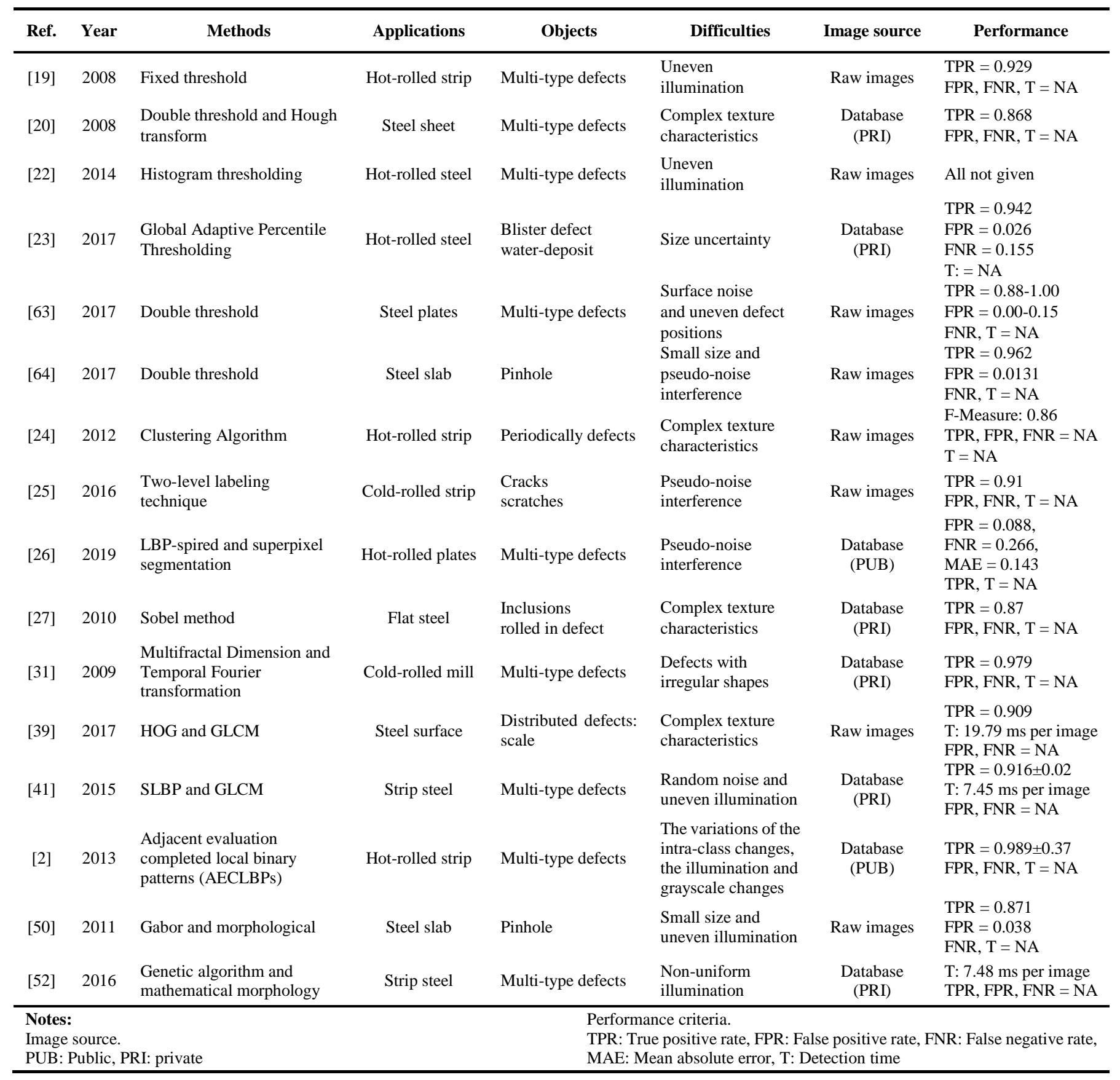

\section{5) Gray-Level Statistic}

Using thresholding methods for defect detection directly may be ineffective in low contrast images, so it is necessary to analyze the distribution of image gray level before threshold operation. Yang et al. [32] utilized the features (i.e., mean value and distribution of pixels) from steel surface background to separate bright and dark defect objects simultaneously. Further, to be insensitive to noise, Choi et al. [33] first estimated the distribution of background by a spectral-based approach and then locally refined the defective regions to obtain the probabilistic estimation. This method is superior to the previous defect detection methods and gives robust results even in noisy environment. However, the above methods for surface defect detection are limited by application scenarios due to the diversity of surface defects. Ma et al. [34] proposed a neighborhood gray-level difference method using the multidirectional gray-level fluctuation which combined the advantages of global and local characteristics. The proposed algorithm not only enhances the generalization also improve the accuracy of surface defects inspection.

\section{6) Co-occurrence Matrix}

Gray level co-occurrence matrix (GLCM) is a common mean to describe texture by studying the spatial correlation of gray level. In 1973, Haralick et al. [35] first presented GLCM, the 
TABLE II

StrengthS and WeaknesSES of Different Statistical Methods of Defect DeteCtion

\begin{tabular}{|c|c|c|}
\hline Taxonomy & Methods & Strengths \\
\hline \multirow{8}{*}{ Statistical } & Thresholding & Simple, easy to understand and implement. \\
\hline & Clustering & Robust to noise and with high computational efficiency. \\
\hline & Edge-based & $\begin{array}{l}\text { Can extracted some low-order features of the image and } \\
\text { easy to realize. }\end{array}$ \\
\hline & Fractal Dimension & $\begin{array}{l}\text { The overall information of images can be expressed by } \\
\text { partial features. }\end{array}$ \\
\hline & Gray-level statistic & Suitable for low resolution images. \\
\hline & Co-occurrence matrix & $\begin{array}{l}\text { The spatial relation of extracted image pixels is } \\
\text { complete and accurate. }\end{array}$ \\
\hline & Local binary pattern & $\begin{array}{l}\text { Can quickly extract discriminative features with rotation } \\
\text { and gray invariance. }\end{array}$ \\
\hline & Morphological & $\begin{array}{l}\text { Highly suitable for random or natural textures and } \\
\text { computationally simple. }\end{array}$ \\
\hline
\end{tabular}

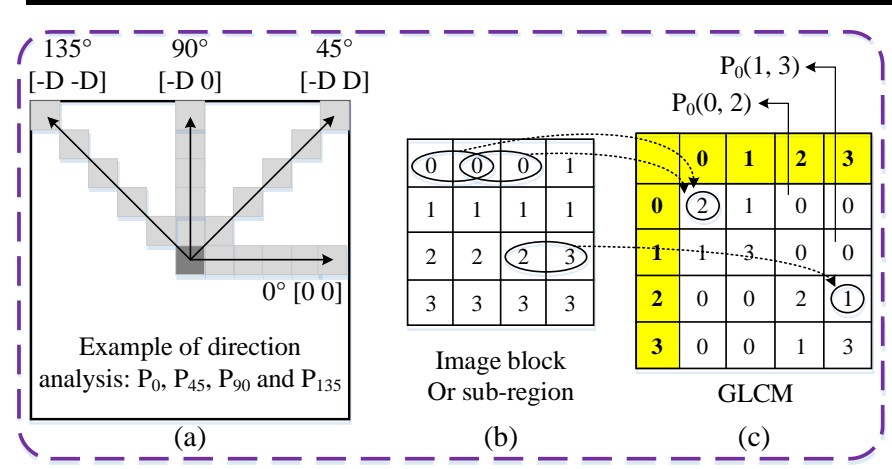

Fig. 5. (a) Direction analysis, (b) an image block and (c) the GLCM of $\mathrm{P}_{0}$.

matrix is defined according to the spatial relation between adjacent pixels of the input image, then based on the GLCM, 14 texture descriptors (i.e., angular second moment, contrast, correlation, entropy, variance, sum of average, sum of variance, inverse difference moment, variance of difference, sum of entropy, difference of entropy, shadow of clustering, prominence of clustering and maximal probability) are generated to successfully describe the relationship between adjacent pixels in an image by calculating the angular relations and distances between adjacent resolution units. Fig. 5 shows the direction analysis of GLCM with a simple example. Subsequently, GLCM has shown powerful ability on automatic texture discrimination in [36-38]. However, it is not an easy job to balance the matrix performance and the window size. In order to overcome the local-descriptive limitation of GLCM, authors in [39] combined complementary feature-sets of the histogram of oriented gradient (HOG) and GLCM to describe global and local textures of steel surface images, respectively. But this approach is sensitive to background noises and ununiform gray level changes, moreover, the computation is relatively complex. Thus Tsai et al. [40] used the weighted eigenvalue of GLCM as a single discriminative feature, so low computational complexity and considerable robustness to noise were achieved simultaneously. Nevertheless, there might be some potential but useful discriminative features in GLCM,

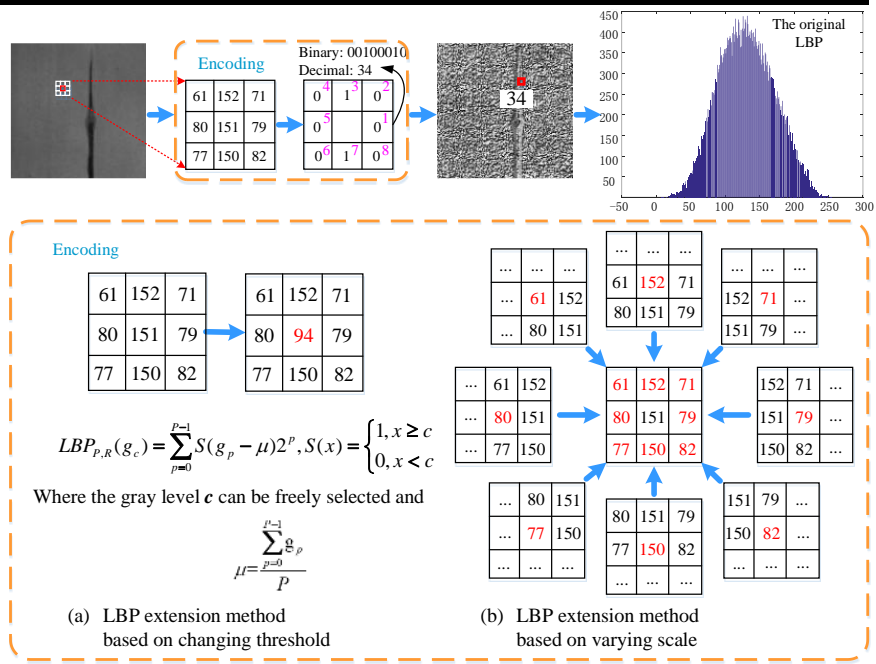

Fig. 6. The standard pipeline of original LBP and the variants of LBP based on changing threshold and scale.

which could be explored for future texture analysis. Furthermore, lots of other types of features extracted by some descriptors are suggested to be fused with those of GLCM, and smoothed local binary pattern (SLBP) [41] is a typical example of this method. If so, more descriptive feature vectors can be built for better surface defect recognition of flat steels.

\section{7) Local Binary Pattern}

As a classical operator, local binary pattern (LBP) is widely used to characterize local texture features of images, which has significant advantages of rotation and gray invariance. In 1994, LBP is first proposed by Ojala et al. in [42], Later, LBP is frequently used to detect defects on flat steel surface [43-45]. In order to overcome the shortcomings of the original LBP (i.e., weak global descriptive and noise-sensitive), various LBP variants are developed based on changing the threshold or scale of the original LBP (refer to Fig. 6), and these variants are widely applied on defect detection of flat steel surface. For example, Wang et al. [26] proposed a LBP-inspired feature extractor by estimating the variations of four directions 
simultaneously, which are horizontal, vertical, and two diagonal directions, so that the features extracted by this method have better visual discrimination. Still, the noise-sensitive has not been eliminated for this method. Song et al. [2] designed an adjacent evaluation completed local binary pattern (AECLBP) by replacing the central pixel with its neighbor pixels. Authors claimed that AECLBP had achieved considerable recognition accuracy and great robustness to noise. However, its scale adaptability is not so preeminent as it inherits the nature of CLBP. Further, Chu et al. [41] proposed a novel LBP version called SLBP, fusing the SLBP frames and GLCM, this method can not only suppress noise effectively, but also extract features with scale, rotation, illumination and translation invariance. Nevertheless, descriptive information among non-uniform patterns have been ignored in all these LBP variants. Using reverse thinking, Luo et al. [3] proposed a generalized completed local binary patterns (GCLBP) by first exploring the non-uniform patterns to supplement the descriptive information in uniform patterns. Further the work of GCLBP, Luo et al. developed a more effective LBP-descriptor (namely SDLBP) in [46], which has remarkable advantages in anti-interference and simplicity of calculation. As a lightweight feature descriptor, LBP variants can be applied on both defect detection and classification, developing more noise-robust and scale-invariant LBP variants or LBP-like descriptors is highly encouraged and coincides with the AVI future trends.

\section{8) Morphological}

Mathematical morphology is an arithmetical tool for image analysis based on morphological structural elements. It has a huge influence on the theory and technology of image processing, especially on shape and structure analysis, which has been widely applied in noise removal [47, 48], feature extraction [49, 50] and image enhancement [51, 52]. Mathematical morphology is specialized in edge processing for its capability of global description. Song et al. [53] removed the edges of oil pollution interference and reflective pseudo-defect by fusing dilation and erosion operations into image subtraction operations. Further, this research team [25] utilized morphology subtraction to extracted defect edges from industrial liquid region on steel surface in the cold rolling process. With the firm and complete theory basis, mathematical morphology is widely used in nearly all aspects in image processing, including image segmentation, feature extraction, edge detection, image filtering, image enhancement, and so on. Nevertheless, the calculation expenses when using morphology should be highly emphasized in the online application of surface defect detection for flat steels, as it mainly relies on a so-called structural element probe to traverse pixels on image for collecting image information, but such operation will generate a large amount of calculation.

\section{9) Brief Summary}

Table I and Table II give a quick glance for these eight types of statistical methods. In summary, these methods are based on two kinds of fundamental structural properties, regularity and local orientation (anisotropy), both properties have great perceived value. Chetverikov et al. [54] analyzed and compared these two approaches comprehensively, then they concludes that the approaches presented above support and complement each other in a natural and understandable way.

\section{B. Spectral}

Although the statistical approaches occupy the largest amount of literatures for steel surface detection in this context, many of them fail to yield reliably correct detection results for several defects with subtle intensity transitions (such as thin roll marks, tiny scratches) especially when illumination varies or pseudo-defect visits frequently. Consequently, emergent AVI methods are highly expected for steel surface defect detection in real-world production. Early report about AVI system for hot steel slabs [55] has recommended that it may be possible to find better solutions in transform domain which are less sensitive to noise and intensity variations than the direct processing methods in pixel domain, which will be reviewed in the following subsections.

\section{1) Fourier Transform}

With the appearance of the Fourier transform (FT), image features of translation invariance, expansion invariance and rotation invariance are realized. Generally, the defect images obtained directly from the steel production line need to be further processed to effectively enhance the quality of images. For removing the background noise, Yazdchi et al. [31] adopted temporal Fourier analysis to eliminate black and white vertical strips in the images formed by the steel plate reflecting ambient light, which appears as the band near a single direct current (DC) term. Similarly, to detect longitudinal cracks from complicated backgrounds on con-casting slab surfaces, Fourier amplitude spectrum of each sub-band is computed to get features with translational invariance [56]. Inspired by discrete Fourier transform, Aiger et al. [57] proposed an unsupervised method based on phase only transform (PHOT), which can persist only irregular patterns to present defects. This novel approach is shown to be effective and generic on various texture surfaces (i.e., wood, steel, ceramic and silicon wafers). Nevertheless, the FT-based approaches are inadequate under the circumstances that Fourier frequency components related to the background and defect areas are highly mixed together. This is because it is difficult to implement non-interference each other during processing frequency domain components associated with background or defect respectively.

\section{2) Gabor Filters}

Fourier transform represents an image by obtaining global features in the frequency domain, thus most of local descriptive information is ignored in the spatial domain. This shortcoming is implicitly but markedly made up by Gabor filters in both the spatial and frequency domains by modulating a specific Gaussian kernel function on a sinusoidal wave with a certain frequency [58]. Then localized and oriented frequency analysis can be achieved by using a simple 2-D Gabor filter[59]. For the targeted task of surface defect detection for flat steels in this paper, Gabor function should be chosen carefully because it significantly affects spatial localization, orientation selectivity and spatial frequency characterization $[60,61]$. This point has 


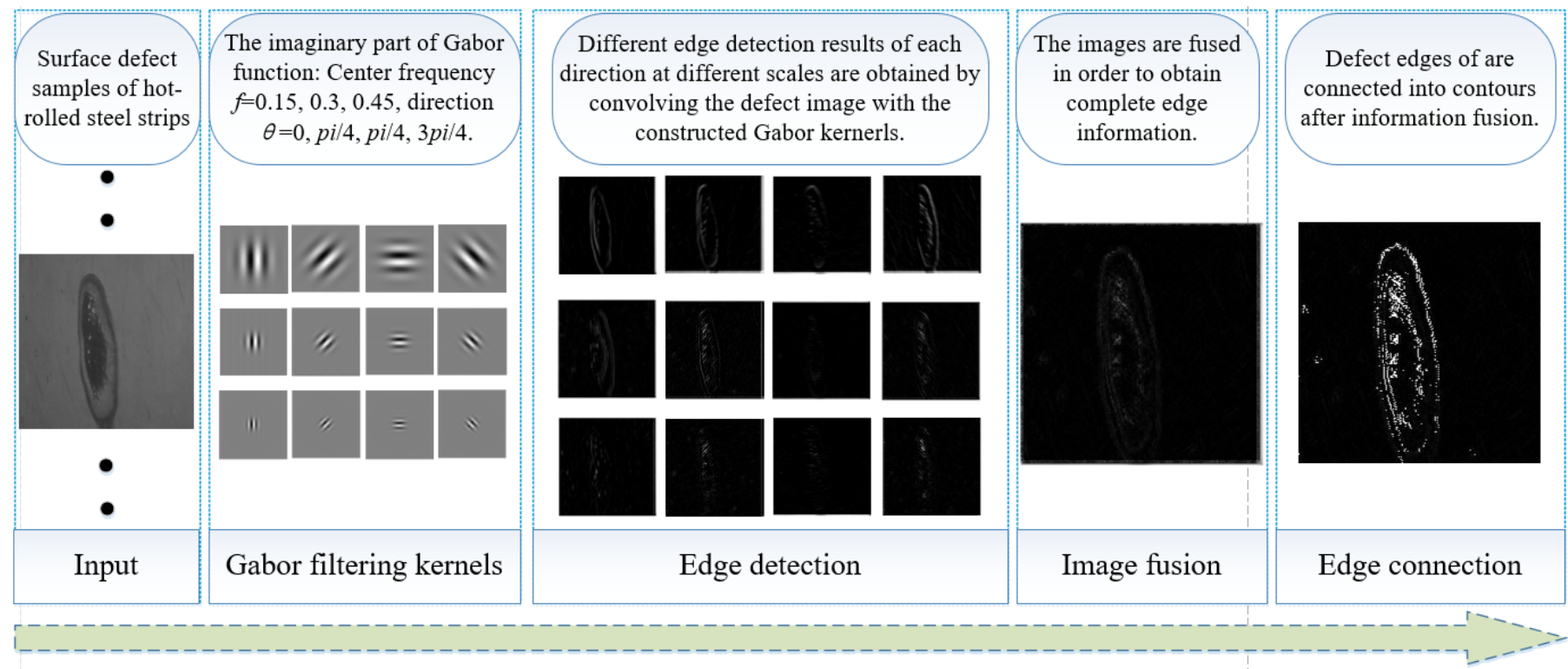

Fig.7. An example of using Gabor filters to detect defect edges.

also been emphasized more than once during the Gabor feature extraction process when it was used for defect detection of flat steel products $[50,62,63]$. It is well recognized that the real and imaginary part can be respectively used for image smoothing and edge detection for a typical Gabor detector. The parameters of Gabor filter are mainly decided by the defect size and direction, it is thus hard to obtain desirable results for miscellaneous defects with various sizes by a single Gabor filter. Accordingly, Choi et al.[64] proposed a two- Gabor-filter combinational method enhanced by morphological features to separate pinholes on steel slabs. Similarly, Medina et al. [65] claimed that the correct defection rate could be increased by fusing Gabor features to other classical image features to a large extent. It was also drawn in [65] that real-time aspect should be attached great importance to on-site application of defect detection for industrial manufacturing. The detection acceleration method by employing Log-Gabor filter bank presented in [66] provides a typical case about this assertion. The above methods have proven that Gabor filtering performs well on characterizing distinctive texture patterns. Besides, Gabor can be combined with statistical methods to get better results (like LBP, GLCM, fractal), and Alvaro et al. [67] confirmed that the combinational approach based on Gabor filter and volumetric fractal dimension possesses promising ability of obtaining rich texture features.

\section{3) Optimized FIR Filters}

The filter optimization process is essentially to effectively separate the frequencies of the defect-free texture with low signal energy and the defective texture with high signal energy [68]. As a typical optimized filter, finite impulse response (FIR) filter provides relatively preeminent feature separation between the defect-free and the defective regions from the FIR-filtered frames [8] . Kumar pointed out in his Ph. D. dissertation [69] that FIR filter performs better both on optimization scale and computational expense than infinite impulse response (IIR) and Gabor filters as FIR filter has more free available turning parameters. Further, Kumar applied FIR filters on the fabric defect detection and obtained milestone achievement in textile industry [70, 71]. Inspired by this trend, Jeon et al. [72] proposed a novel sub-optimal FIR filtering scheme that adaptively combines optimized FIR filters by considering the texture features of images captured from a dual-light switching-lighting device, to detect various shapes of defects on steel surfaces. This innovative detection method is effective to handle non-uniform surfaces and scale-oxidized substances caused during the hot-working manufacturing process. In addition, FIR filters are very suitable to be embedded in FPGAs, which is compliant with the lightweight trend of the instrumentation and measurement society. To sum up, optimized FIR filtering shows enormous application potentiality in the detection of defects for flat steel surfaces.

\section{4) Wavelet Transform}

Compared with Gabor filters, wavelet transform can not only move the time-frequency window, but also automatically adjust the window with the change of the frequency in the center of the window. Meanwhile, the characteristics of wavelet are more in line with human visual mechanism. Consequently, wavelet transform can effectively extract information from signals and perform multi-scale analysis of functions or signals through scaling and shifting operations. Due to the existence of pseudo defects caused by water droplets, oxidized scales, uneven illumination and so on, the defect detection of steel surface becomes increasingly challenging. Five different types of wavelets, namely, Haar, Daubechies 2 (DB2), Daubechies 4 (DB4), biorthogonal spline (Bior), and multiwavelet, have been evaluated by Ghorai et al. [1] to extract features of small-size image blocks. However, the anti-noise measure resisting the uneven illumination is absent in this scheme. Yan et al. [73] proposed a novel wavelet-based image filtering algorithm based on anisotropic diffusion. The features of anisotropic diffusion encouraging the intra-region smoothing adaptively and inhibiting the inter-region diffusion permit that wavelet anisotropic diffusion method can not only extract defect from noisy backgrounds reliably, but also can separate high and low 
TABLE III

List of Some of Typical Spectral Methods of Defect Detection

\begin{tabular}{|c|c|c|c|c|c|c|c|}
\hline Ref. & Year & Methods & Applications & Objects & Difficulties & Image source & Performance \\
\hline$[31]$ & 2009 & $\begin{array}{l}\text { Multifractal } \\
\text { Dimension and } \\
\text { Temporal Fourier } \\
\text { transformation }\end{array}$ & Cold-rolled mill & Multi-type defects & $\begin{array}{l}\text { Defects with irregular } \\
\text { shapes }\end{array}$ & $\begin{array}{l}\text { Database } \\
\text { (PRI) }\end{array}$ & $\begin{array}{l}\mathrm{TPR}=0.979 \\
\text { FPR, FNR, T = NA }\end{array}$ \\
\hline$[56]$ & 2013 & Fourier transform & $\begin{array}{l}\text { Continuous casting } \\
\text { slabs }\end{array}$ & $\begin{array}{l}\text { Longitudinal } \\
\text { cracks }\end{array}$ & $\begin{array}{l}\text { Complex texture } \\
\text { characteristics }\end{array}$ & Raw images & $\begin{array}{l}\text { TPR }=0.919 \\
\text { FPR }=0.0893 \\
\text { FNR, T = NA }\end{array}$ \\
\hline$[50]$ & 2011 & $\begin{array}{l}\text { Gabor and } \\
\text { morphological }\end{array}$ & Steel slab & Pinhole & $\begin{array}{l}\text { Small size and uneven } \\
\text { illumination }\end{array}$ & Raw images & $\begin{array}{l}\text { TPR }=0.871 \\
\text { FPR }=0.038 \\
\text { FNR, T = NA }\end{array}$ \\
\hline$[62]$ & 2015 & Gabor filtering & Thick plates & Periodic defects & $\begin{array}{l}\text { Non-uniform } \\
\text { illumination }\end{array}$ & Raw images & $\begin{array}{l}\mathrm{TPR}=1.00 \\
\mathrm{FPR}=0.0075 \\
\mathrm{FNR}, \mathrm{T}=\mathrm{NA}\end{array}$ \\
\hline$[63]$ & 2017 & Double threshold & Steel plates & Multi-type defects & $\begin{array}{l}\text { Surface noise and } \\
\text { uneven defect } \\
\text { positions }\end{array}$ & Raw images & $\begin{array}{l}\text { TPR }=0.88-1.00 \\
\text { FPR }=0.00-0.15 \\
\text { FNR, T = NA }\end{array}$ \\
\hline$[64]$ & 2017 & Double threshold & Steel slab & Pinhole & $\begin{array}{l}\text { Small size and } \\
\text { pseudo-noise } \\
\text { interference }\end{array}$ & Raw images & $\begin{array}{l}\mathrm{TPR}=0.962 \\
\mathrm{FPR}=0.0131 \\
\mathrm{FNR}, \mathrm{T}=\mathrm{NA}\end{array}$ \\
\hline$[66]$ & 2011 & Log Gabor filter bank & Flat surfaces & $\begin{array}{l}\text { Products on } \\
\text { homogeneous flat } \\
\text { surface }\end{array}$ & $\begin{array}{l}\text { Complex texture } \\
\text { characteristics }\end{array}$ & $\begin{array}{l}\text { Database } \\
\text { (PUB) }\end{array}$ & $\begin{array}{l}\mathrm{TPR}=0.998 \\
\mathrm{FPR}, \mathrm{FNR}, \mathrm{T}=\mathrm{NA}\end{array}$ \\
\hline [72] & 2015 & $\begin{array}{l}\text { Optimized } \\
\text { general-finite } \\
\text { impulse-response filter }\end{array}$ & Steel plate & Multi-type defects & $\begin{array}{l}\text { Non-uniform } \\
\text { brightness and various } \\
\text { shaped defects }\end{array}$ & $\begin{array}{l}\text { Database } \\
\text { (PRI) }\end{array}$ & $\begin{array}{l}\text { TPR = } 0.979 \\
\text { T: } 106 \text { ms per frame } \\
\text { FPR, FNR = NA }\end{array}$ \\
\hline$[1]$ & 2012 & $\begin{array}{l}\text { Wavelet feature sets } \\
\text { and VVRKFA }\end{array}$ & Hot-rolled steel & Multi-type defects & $\begin{array}{l}\text { Large surface, } \\
\text { variation in } \\
\text { appearance, and their } \\
\text { rare occurrences }\end{array}$ & $\begin{array}{l}\text { Database } \\
\text { (PRI) }\end{array}$ & $\begin{array}{l}\text { G-mean: } 93.8 \% \text {, } \\
\text { F-measure: } 90.4 \% \\
\text { T: } 86.5 \text { ms per image } \\
\text { TPR, FPR, FNR = NA }\end{array}$ \\
\hline$[73]$ & 2014 & $\begin{array}{l}\text { A novel wavelet-based } \\
\text { image filtering } \\
\text { algorithm }\end{array}$ & Cold-rolled strip & Multi-type defects & $\begin{array}{l}\text { The complexity of } \\
\text { surface texture }\end{array}$ & All not given & All not given \\
\hline$[74]$ & 2008 & $\begin{array}{l}\text { Undecimated Wavelet } \\
\text { Transform }\end{array}$ & Hot-rolled plates & Horizontal crack & $\begin{array}{l}\text { Pseudo-noise } \\
\text { interference and } \\
\text { uneven illumination }\end{array}$ & $\begin{array}{l}\text { Database } \\
\text { (PRI) }\end{array}$ & $\begin{array}{l}\mathrm{TPR}=0.902 \\
\text { FPR, FNR, T = NA }\end{array}$ \\
\hline$[75]$ & 2014 & $\begin{array}{l}\text { Cascading wavelet } \\
\text { transforms }\end{array}$ & Hot-rolled strip & Multi-type defects & Local deformation & $\begin{array}{l}\text { Database } \\
\text { (PUB) }\end{array}$ & $\begin{array}{l}\mathrm{TPR}=0.986 \pm 0.59 \\
\mathrm{FPR}, \mathrm{FNR}, \mathrm{T}=\mathrm{NA}\end{array}$ \\
\hline$[78]$ & 2012 & Bandelet-PCNN & Strip steel & Multi-type defects & $\begin{array}{l}\text { Pseudo-Gibbs } \\
\text { phenomena around } \\
\text { singularities }\end{array}$ & All not given & All not given \\
\hline [79] & 2015 & Shearlet transform & $\begin{array}{l}\text { Continuous casting } \\
\text { slabs, hot-rolled steels, } \\
\text { and aluminum sheets }\end{array}$ & Multi-type defects & $\begin{array}{l}\text { Complicated } \\
\text { background, } \\
\text { pseudo-defects } \\
\text { interference, } \\
\text { low contrast and small } \\
\text { size }\end{array}$ & $\begin{array}{l}\text { Database } \\
\text { (PRI) }\end{array}$ & $\begin{array}{l}\text { TPR }=0.944,0.956 \\
\text { and } 0.925, \text { for three } \\
\text { types of flat steel } \\
\text { FPR, FNR, T = NA }\end{array}$ \\
\hline$[80]$ & 2018 & RNAMlet & $\begin{array}{l}\text { Continuous casting } \\
\text { slabs, hot rolled steel } \\
\text { plates and cold rolled } \\
\text { steel strips }\end{array}$ & Multi-type defects & $\begin{array}{l}\text { Different surface } \\
\text { appearances and } \\
\text { different speeds of } \\
\text { movement }\end{array}$ & $\begin{array}{l}\text { Database } \\
\text { (PRI) }\end{array}$ & $\begin{array}{l}\mathrm{TPR}=0.885,0.972 \\
\text { and } 0.984, \text { for three } \\
\text { types of flat steel } \\
\text { FPR, FNR, T = NA }\end{array}$ \\
\hline
\end{tabular}

Notes:

Image source.

PUB: Public, PRI: private

frequency components effectively. Similarly, Wu et al. [74] proposed an undecimated wavelet transform (UMT) for solving the problem of false alarms resulted from oxidized scales and water marks with an overall recognition rate of $90.23 \%$. Besides the challenge of pseudo defects, some steel surface defects produce very subtle intensity transitions. Song et al. [75] employed a scattering convolution network (SCN) based on wavelet transform to improve the tolerance ability on local and linearized deformations. This method has been successfully
Performance criteria.

TPR: True positive rate, FPR: False positive rate, FNR: False negative rate, MAE: Mean absolute error, T: Detection time

applied on surface defect detection for hot-rolled strips and obtained an average correct recognition accuracy of $97.22 \%$.

\section{5) Multiscale Geometric Analysis}

The singularity of 2-D defect images captured from steel production lines is primarily depicted by edge information which appears as irregular lines or surfaces. Wavelet transform can optimally characterize the point singularity but can hardly characterize lines and surface singularities due to the finiteness of separable wavelet directions. An appropriate solution to 
TABLE IV

StRENGTHS AND WeaKnESSES of DifFERENT SPeCtral Methods of DefECt DeteCtion

\begin{tabular}{|c|c|c|c|}
\hline Taxonomy & Methods & Strengths & Weaknesses \\
\hline \multirow{6}{*}{ Spectral } & Fourier Transform & Invariance to translation, expansion and rotation. & $\begin{array}{l}\text { Difficult to realize non-interference when } \\
\text { dealing with frequency-domain components } \\
\text { related to background or defect. }\end{array}$ \\
\hline & Gabor Filters & $\begin{array}{l}\text { Suitable for high dimensional feature space with low computational } \\
\text { burden. }\end{array}$ & $\begin{array}{l}\text { Hard to determine the optimal filter } \\
\text { parameters and no rotation invariance. }\end{array}$ \\
\hline & $\begin{array}{l}\text { Optimized FIR } \\
\text { Filters }\end{array}$ & $\begin{array}{l}\text { Suitable for defects with subtle intensity variation and more free } \\
\text { parameters to keep the computational simplicity. }\end{array}$ & $\begin{array}{l}\text { Limitations to solve the problem of low } \\
\text { frequency. }\end{array}$ \\
\hline & Wavelet Transform & $\begin{array}{l}\text { Suitable for multi-scale image analysis and can compression image } \\
\text { efficiently with less information loss. }\end{array}$ & $\begin{array}{l}\text { Easily to be affected by feature correlations } \\
\text { between the scales. }\end{array}$ \\
\hline & $\begin{array}{c}\text { Multiscale } \\
\text { Geometric Analysis }\end{array}$ & $\begin{array}{l}\text { Suitable for the optimal and sparse representation of high-dimension } \\
\text { data. Good at image processing of strong noise background. }\end{array}$ & Exist redundancy problem. \\
\hline & Hough Transform & Strong anti-interference ability and insensitive to noise. & $\begin{array}{l}\text { Only detects defects of specific shapes (i.e., } \\
\text { lines, circles and ellipses). }\end{array}$ \\
\hline
\end{tabular}

this problem is to employ multiscale geometric analysis (MGA) whose multi-directivity renders protection and detection of edge features (especially singular edges) more precisely. Generally, MGA methods are separated into adaptive and non-adaptive types. The adaptive methods are represented by Bandelet [76] and Tetrolet [77]. Zhang et al. [78] have proposed an image fusion method based on Bandelet-PCNN (Pulse coupled neural networks) model to solve the problem of the pseudo-Gibbs phenomena around singularities. For quality assurance of con-casting slabs and hot strips, $\mathrm{Xu}$ et al. successively proposed a Shearlet-based feature extraction method (DST-KLPP) [79] and an adaptive MGA method (RNAMlet) [80], both of them emphasized much on detection rates and computation expenses. When it comes to the typical non-adaptive MGA such as Ridgelet [81] and Curvelet [82], Ai et al. [56] applied Curvelet enhanced by kernel locality preserving projections to track longitudinal cracks on con-casting slabs. Nevertheless, how to effectively distinguish confused defect edges and active background textures is still an open research topic for both engineering and academia.

\section{6) Hough Transform}

Hough transform (HT) [83] is considered as a powerful tool in well-defined line-feature detection. Its applications can be found in fingerprint identification $[84,85]$ and vehicle license plate recognition [86]. Interestingly, Sharifzadeh et al. [20] applied HT to detect defects of holes, scratches, coil breaks and rusts on cold-rolled steel strips. However, it is difficult to raise the correct detection rates to more than $90 \%$. Hough line detection has the advantage of strong anti-interference ability and is also insensitive to noises, incomplete part of edges, and other coexisting non-linear structures. However, HT can only track the direction of edges, the length information of line segment is lost. It is worth noting that the time and space complexity should be effectively reduced if using HT for surface defect detection of flat steels.

\section{7) Brief Summary}

Table III and Table IV give a quick glance for these six types of spectral methods, the advantages and disadvantages are also analyzed briefly. In general, spectral methods are dedicated to find a special transform domain where the defect objects can be more easily and completely separated from the both the local and global backgrounds.

\section{Model-based}

Naturally, statistical-based methods are relatively sensitive to noise while spectral-based methods lack local information, both of them have bottlenecks on representing miscellaneous defects and stochastic background variations appeared on textured surfaces. Model-based methods tend to perform better for diverse defect detection by projecting original texture distribution of image blocks to low-dimensional distribution via a structurally special model enhanced by parameter learning. Several model-based methods are now briefly discussed below.

\section{1) Markov Random Field Model}

In 1983, on the basic idea of that a texture has interaction among relevant random variables in a stochastic or periodic 2-D field, Cross et al. [87] first used markov random field (MRF) as texture model, the structure of 2-D MRF can well represent the spatial correlation of image pixels. Inspired by this concept, Gayubo et al. [88] utilized MRF to restore flat steel defects (i.e., cracks) and eliminate the spurious features (i.e., pseudo). Further, Xu et al. [89] dramatically decreased the detection false rate from $18.8 \%$ to $3.7 \%$ by using the proposed context-adaptive hidden Markov tree model (CAHMT) based on an assertion that the correlation of wavelet coefficients of flat steel surface images at different scales satisfies Markov property. The recent literatures exhibit the huge application potentiality of MRF on industrial surface defect detection.

\section{2) Weibull Model}

Some flat steel surface defects that produce subtle intensity transitions may be difficult to be detected by using the above MRF-based method. A potential solution to handle the detection task of such defects is to utilize the relatively complete descriptive superiorities on texture contrast, scale, and shape of Weibull distribution [90]. Continue this idea, Fofi et al. [16] proposed a novel, non-parametric and efficient Weibull-based defect detection method by computing two parameters of a Weibull fit for the distribution of image gradients in local regions. This unsupervised method performs 


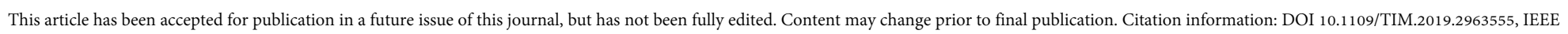
Transactions on Instrumentation and Measurement

TABLE V

List of Some of Typical Model-BASEd Methods of DefeCt DeteCtion

\begin{tabular}{|c|c|c|c|c|c|c|c|}
\hline Ref. & Year & Methods & Applications & Objects & Difficulties & Image source & Performance \\
\hline [88] & 2006 & $\begin{array}{l}\text { Markov random field } \\
\text { model }\end{array}$ & Sheet-metal & Split defects & $\begin{array}{l}\text { Remaining spurious } \\
\text { features }\end{array}$ & All not given & All not given \\
\hline [89] & 2013 & Hidden Markov tree & Steel strips & Multi-type defects & $\begin{array}{l}\text { Complex texture } \\
\text { characteristics }\end{array}$ & $\begin{array}{l}\text { Database } \\
\text { (PRI) }\end{array}$ & $\begin{array}{l}\text { TPR }=0.944 \\
\text { FNR }=0.037 \\
\text { FPR, T = NA }\end{array}$ \\
\hline [16] & 2013 & Weibull distribution & Steel surface & Multi-type defects & $\begin{array}{l}\text { Arbitrary deviations of } \\
\text { the reference texture }\end{array}$ & $\begin{array}{l}\text { Database } \\
\text { (PRI) }\end{array}$ & $\begin{array}{l}\text { EER: } 3.2 \%, \\
\text { AUC }=0.99 \\
\text { FNR }=0.051 \\
\text { TPR, FPR, T = NA }\end{array}$ \\
\hline [91] & 2017 & $\begin{array}{l}\text { Haar-Weibull-Variance } \\
\text { model }\end{array}$ & Steel strips & Multi-type defects & $\begin{array}{l}\text { Miscellaneous patterns, } \\
\text { low contrast and } \\
\text { Pseudo-noise interference }\end{array}$ & $\begin{array}{l}\text { Database } \\
\text { (PRI) }\end{array}$ & $\begin{array}{l}\text { TPR }=0.962 \\
\text { T: } 52 \text { ms per image } \\
\text { FPR, FNR, T = NA }\end{array}$ \\
\hline [94] & 2013 & $\begin{array}{l}\text { Saliency convex active } \\
\text { contour model }\end{array}$ & $\begin{array}{l}\text { Silicon Steel } \\
\text { Strip }\end{array}$ & $\begin{array}{l}\text { Spot-defect } \\
\text { Steel-pit-defect }\end{array}$ & $\begin{array}{l}\text { Micro defects in the } \\
\text { cluttered background }\end{array}$ & All not given & All not given \\
\hline [95] & 2018 & Active contour model & $\begin{array}{l}\text { Large steel } \\
\quad \text { roller }\end{array}$ & $\begin{array}{l}\text { Speckles } \\
\text { Chatter marks } \\
\text { Feed traces }\end{array}$ & $\begin{array}{l}\text { Large dimension and } \\
\text { weight }\end{array}$ & All not given & All not given \\
\hline [99] & 2018 & $\begin{array}{l}\text { Double Low-Rank and } \\
\text { Sparse Decomposition } \\
\text { Model }\end{array}$ & Hot-rolled strip & Multi-type defects & $\begin{array}{l}\text { Noise and uneven } \\
\text { illumination }\end{array}$ & $\begin{array}{l}\text { Database } \\
\text { (PUB) }\end{array}$ & $\begin{array}{l}\text { AUC: } 0.8350 \text {, } \\
\text { MAE: } 0.1584 \text {, } \\
\text { T: } 171.3 \text { ms per image } \\
\text { TPR, FPR, FNR = NA }\end{array}$ \\
\hline [26] & 2019 & Compact model & $\begin{array}{l}\text { Hot-rolled } \\
\text { plates }\end{array}$ & Multi-type defects & $\begin{array}{l}\text { The interference of } \\
\text { pseduo-defects }\end{array}$ & $\begin{array}{l}\text { Database } \\
\text { (PUB) }\end{array}$ & $\begin{array}{l}\mathrm{FPR}=0.088 \\
\mathrm{FNR}=0.266 \\
\mathrm{MAE}=0.143 \\
\mathrm{TPR}, \mathrm{T}=\mathrm{NA}\end{array}$ \\
\hline [17] & 2019 & $\begin{array}{l}\text { A unique guidance } \\
\text { template }\end{array}$ & Steel strips & Multi-type defects & $\begin{array}{l}\text { Defects with } \\
\text { miscellaneous patterns }\end{array}$ & $\begin{array}{l}\text { Database } \\
\text { (PRI) }\end{array}$ & $\begin{array}{l}\text { TPR }=0.962 \\
\text { T: } 35 \text { ms per image } \\
\text { FPR, FNR = NA }\end{array}$ \\
\hline
\end{tabular}

Notes:

Image source.

PUB: Public, PRI: private
Performance criteria. TPR: True positive rate, FPR: False positive rate, FNR: False negative rate, EER: Equal error rate, AUC: Area under curve, MAE: Mean absolute error, T: Detection time

TABLE VI

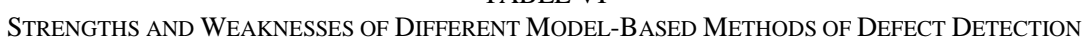

\begin{tabular}{lcll}
\hline Taxonomy & Methods & \multicolumn{1}{c}{ Strengths } & Weaknesses \\
\hline \multirow{2}{*}{ Markov Random Field Model } & $\begin{array}{l}\text { Can be combined with statistical and spectral } \\
\text { methods for segmentation applications to } \\
\text { capture the local texture orientation information. }\end{array}$ & $\begin{array}{l}\text { Cannot detect small defects. Not suitable for global } \\
\text { texture analysis. Strong spatial constraint. }\end{array}$ \\
Model-based & Weibull model & $\begin{array}{l}\text { Has superiorities on describing the contrast, } \\
\text { scale and shape of textures. }\end{array}$ & $\begin{array}{l}\text { Hard to detect defects with gradual intensity or with } \\
\text { low contrast. }\end{array}$ \\
& Active contour model achieve sub-pixel accuracy of object & $\begin{array}{l}\text { Can } \\
\text { boundaries. Has good performance on both } \\
\text { spot-defect and steel-pit-defect. }\end{array}$ & $\begin{array}{l}\text { Hard to calculate the convergence position due to } \\
\text { lacking constraints. }\end{array}$ \\
\hline
\end{tabular}

well on a large industrial optical inspection database, where involves some highly challenging flat steel defects. However, it is hard for Weibull distribution to handle defects with gradual intensity or with low contrast. Hence, Liu et al. [91] developed a Haar-Weibull-variance (HWV) model by replacing the features of local gradient magnitude by Haar features from local patches. This method is reported to have achieved an average correct detection rate of $96.2 \%$ on a homogeneously textured defect dataset gathered from an actual hot-rolling mill.

\section{3) Active Contour Model}

The basic idea of active contour model (ACM) is to use continuous curve to express and locate the edge of object (here is, defect) by curve evolution. ACM is popular in image segmentation as it can achieve sub-pixel accuracy of object boundaries [92, 93]. Song et al. [94] proposed saliency convex active contour model (SCACM) by fusing visual saliency map into convex energy minimization function to detect micro surface defects on silicon steel strips. The SCACM yielded good performance on both spot-defect and steel-pit-defect as the fused visual saliency map highlights the potential defects and suppresses the clutter background as well. Yang et al. [95] developed a ACM-based defect detection method without edges through incorporating a variable penalty term and a convolution kernel, authors reported that it can effectively segment defect features with inhomogeneous boundaries from complicated surface textures. The iteration steps and computing time increasingly attract the attention of scholars. 


\section{4) Other Latest Reported Model-based}

There are some latest reported model-based defect detection methods. Susan et al. [96] proposed Gaussian mixture entropy model for defect detection, which is specialized in identifying miscellaneous defects such as holes and stains. Based on low-rank representation, Yan et al. [97] utilized smooth-sparse decomposition (SSD) model for anomaly detection in images, Huangpeng et al. [98] proposed a novel weighted low-rank reconstruction model for automatic visual defect detection, and Zhou et al. [99] presented a double low-rank and sparse decomposition (DLRSD) model to obtain the defective region of steel sheet surface. These approaches are reported to perform well. Wang et al. [26] constructed a compact model to be regarded as a kind of guidance information by mining intrinsic image priors, it offers a good generalization ability for different detection tasks and is sufficiently robust to noise. Further, Wang et al. [17] proposed a guidance template-based defect detection method for strip steel surfaces by introducing a sorting operation to sort gray levels with each column of test image and then subtracts the sorted test image with guidance template to locate defects conveniently, It achieved an average detection rate of $96.2 \%$ on a dataset with 1500 test images involving challenges of uneven illumination. Any information descriptive models with low computational complexity can be considered for the task of surface defect detection for flat steels in future.

\section{5) Brief Summary}

Table V highlights some representatives of model-based detection methods, and the strengths and weaknesses are also gathered in Table VI. In this branch direction, how to found noise robust, theoretically explainable, computationally simple models to adaptively absorb sparse features of defects will attract increasing attentions from both academia and industry.

\section{Machine Learning}

The essence of machine learning is to analyze and learn data then make decisions or predictions accurately for the further operation. With the popularity of artificial intelligence in recent years, machine learning, a powerful branch of model-based methods, have been proposed extensively for defect detection of flat steel surface. As shown in Fig. 8, the defect detection task is essentially handled as a binary (defective or defect-free) classification problem in machine learning methods (or we can call them advanced classifiers). And the machine learning defect detection methods are reviewed in three categories of supervised-, unsupervised- and reinforcement learning.
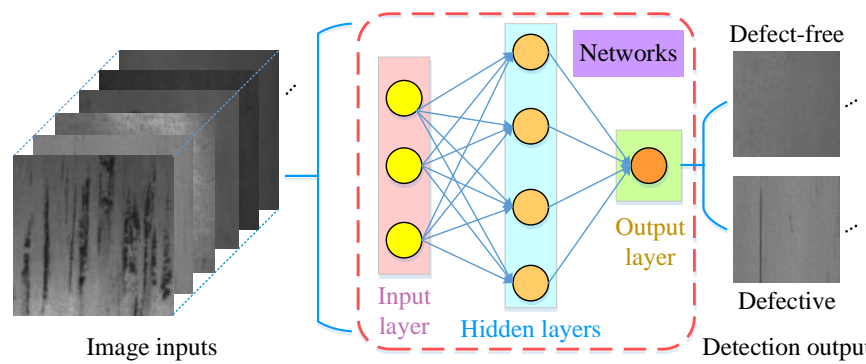

Image inputs

Fig. 8. The general flow of machine learning methods.

\section{1) Supervised Learning}

The goal of supervised learning is to model a conditional distribution between input vectors (surface images) and target vectors (defect label 0 or 1). Support vector machine (SVM), decision trees and neural network are classical examples in this category. As a generalized linear classifier for binary classification of data, SVM is frequently utilized to identify defective and defect-free regions [100, 101]. Ghorai et al. [1] hold that the performance of classifiers in defect detection depends on the feature and classifier combination. The authors fused the classifiers (i.e., SVM and VVRKFA) with different feature sets (i.e., Haar, DB2, DN4) to divide the test images into defective and normal ones, finding that the performance of VVRKFA with one-level Haar features ranks first among all the feature-classifier combinations. The neural network can learn the pattern from the training dataset, and determine the category of the new data according to the previous knowledge. Liu et al. [102] used a two-layer feed-forward neural network to classify the pixel of test images into defect and defect-free regions on the basic idea that the defect detection task is actually a binary classification problem. But a great quantity of parameters of neural networks lead to huge computational complexity. Convolution and subsampling in convolution neural network (CNN) effectively reduce the model size by tailoring the model parameters. Thus, CNN-based architectures are widely applied on automatic feature extraction [103] as well as on image defect detection [104-108] in industrial inspection. For example, Cha et al. [105] proposed a deep CNN to detect cracks on concrete and steel surface without calculating defect features. The framework can effectively resist the interferences caused by the extensively varying real-world situations. This team also designed a structural visual inspection method based on faster region-based $\mathrm{CNN}$ (faster R-CNN) to ensure quasi real-time simultaneous detection of multiple types of defects [109]. Moreover, Song et al. [108] realized precise detection of weak scratches on metal surface by confusing deep CNN and skeleton extraction, the experimental results indicate its strong robustness to background noises. In order to enable CNN-based detection methods to be applied in real-time industrial scenes, an impressive method called you only look once (YOLO) network was proposed by addressing the bi-classification task as a regression problem. Li et al. [110] improved the YOLO network by making it all convolutional and then applied the YOLO-variant to detect surface defects of flat steel. And this network reached $99 \%$ correct detection rate with a speed of 83 FPS on a dataset of 4655 defect images of cold-rolled steel surface. The satisfactory detection performance of supervised learning methods is achieved only with a premise of having a great quantity of labeled image samples on defect database. While collecting and labelling a great number of image samples on industrial manufacturing line are quite labor-intensive and time-consuming, or even to say, impracticable.

\section{2) Unsupervised Learning}

Automated defect detection has always been a challenging task especially in actual industrial application. It is not always easy to gather a large number of labeled image samples, that is, 


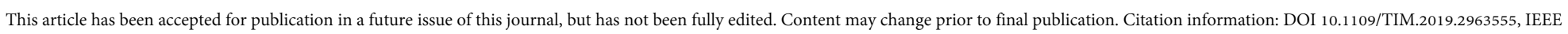
Transactions on Instrumentation and Measurement

TABLE VII

List of Some of Typical Machine Learning Methods of Defect Detection

\begin{tabular}{|c|c|c|c|c|c|c|c|}
\hline Ref. & Year & Methods & Applications & Objects & Difficulties & Image source & Performance \\
\hline [100] & 2014 & Gabor filtering and SVM & Steel plates & Seam cracks & $\begin{array}{l}\text { Small size and low } \\
\text { contrast }\end{array}$ & Raw images & $\begin{array}{l}\mathrm{TPR}=0.945 \\
\mathrm{FNR}=0.003 \\
\mathrm{FPR}, \mathrm{T}=\mathrm{NA}\end{array}$ \\
\hline$[1]$ & 2012 & $\begin{array}{l}\text { wavelet feature sets and } \\
\text { VVRKFA }\end{array}$ & Hot-rolled steel & Multi-type defects & $\begin{array}{l}\text { Various appearance } \\
\text { and rare occurrences }\end{array}$ & $\begin{array}{l}\text { Database } \\
\text { (PRI) }\end{array}$ & $\begin{array}{l}\text { G-mean: } 93.8 \% \text {, } \\
\text { F-measure: } 90.4 \% \\
\text { T: } 86.5 \mathrm{~ms} \text { per image } \\
\text { TPR, FPR, FNR = NA }\end{array}$ \\
\hline$[102]$ & 2005 & $\begin{array}{l}\text { Algorithm based on } \\
\text { feed-forward neural } \\
\text { network }\end{array}$ & Cold-rolled strip & Scratches & $\begin{array}{l}\text { Complex texture } \\
\text { characteristics }\end{array}$ & All not given & All not given \\
\hline$[105]$ & 2017 & A deep $\mathrm{CNN}$ & Steel surface & Cracks & $\begin{array}{l}\text { Extensively varying } \\
\text { real-world situations }\end{array}$ & Raw images & $\begin{array}{l}\mathrm{TPR}=0.974 \\
\text { FPR, FNR, T= NA }\end{array}$ \\
\hline$[106]$ & 2019 & $\begin{array}{l}\text { Classification priority } \\
\text { network }\end{array}$ & $\begin{array}{l}\text { Hot-rolled steel plates, } \\
\text { Hot-rolled steel strips }\end{array}$ & Multi-type defects & $\begin{array}{l}\text { The different } \\
\text { morphological } \\
\text { characteristics of the } \\
\text { same type of defects }\end{array}$ & $\begin{array}{l}\text { Database } \\
\text { (PRI) }\end{array}$ & $\begin{array}{l}\mathrm{TPR}=0.94 \text { and } 0.96 \\
\text { respectively } \\
\text { FPR, FNR, T= NA }\end{array}$ \\
\hline$[107]$ & 2019 & $\begin{array}{l}\text { CNN and long } \\
\text { short-term memory } \\
\text { (LSTM) }\end{array}$ & Steel Plates & Roll marks & $\begin{array}{l}\text { Low contrast in their } \\
\text { background }\end{array}$ & $\begin{array}{l}\text { Database } \\
\text { (PRI) }\end{array}$ & $\begin{array}{l}\text { TPR }=0.862 \\
\text { TPR, FPR, FNR = NA }\end{array}$ \\
\hline$[108]$ & 2019 & $\begin{array}{l}\text { Deep convolutional } \\
\text { neural networks } \\
\text { (DCNNs) }\end{array}$ & $\begin{array}{l}\text { Metal component } \\
\text { surfaces }\end{array}$ & $\begin{array}{l}\text { Weak } \\
\text { micro-scratch }\end{array}$ & $\begin{array}{l}\text { Non-uniform gray } \\
\text { distribution, various } \\
\text { shapes, low contrast } \\
\text { in their background }\end{array}$ & $\begin{array}{l}\text { Database } \\
\text { (PRI) }\end{array}$ & $\begin{array}{l}\text { IoU }=0.8125 \\
\text { TPR, FPR, FNR = NA } \\
T=\text { NA }\end{array}$ \\
\hline$[110]$ & 2018 & $\begin{array}{l}\text { Improved YOLO } \\
\text { detection network }\end{array}$ & Cold-rolled strip & Multi-type defects & $\begin{array}{l}\text { Diverse and complex } \\
\text { features }\end{array}$ & $\begin{array}{l}\text { Database } \\
\text { (PRI) }\end{array}$ & $\begin{array}{l}\text { MAP: } 97.55 \% \\
\text { Recall rate: } 95.86 \% \\
\text { Speed: } 83 \text { FPS } \\
\text { TPR, FPR, FNR = NA } \\
\text { T = NA } \\
\text { TPR }=0.8834\end{array}$ \\
\hline [112] & 2017 & AnoGAN & Multi-type & Multi-type defects & $\begin{array}{l}\text { Small labeled } \\
\text { samples }\end{array}$ & $\begin{array}{l}\text { Database } \\
\text { (PRI) }\end{array}$ & $\begin{array}{l}\text { Recall =0.7277 } \\
\text { AUC }=0.89 \\
\text { TPR, FPR, FNR = NA } \\
\text { T = NA }\end{array}$ \\
\hline$[18]$ & 2019 & $\begin{array}{l}\text { Convolutional } \\
\text { auto-encoder (CAE) }\end{array}$ & Hot-rolled strip & Multi-type defects & $\begin{array}{l}\text { Wide variety of } \\
\text { forms and various } \\
\text { classes }\end{array}$ & $\begin{array}{l}\text { Database } \\
\text { (PUB) }\end{array}$ & All not given \\
\hline$[113]$ & 2018 & GAN and autoencoder & Multi-type & Multi-type defects & $\begin{array}{l}\text { Hard to collect } \\
\text { samples beforehand } \\
\text { and manual labelling } \\
\text { is time-consuming } \\
\text { Collecting and }\end{array}$ & $\begin{array}{l}\text { Database } \\
\text { (PRI) }\end{array}$ & $\begin{array}{l}\text { TPR }=0.985 \\
\mathrm{~T}: 80.3 \mathrm{~ms} \text { per image } \\
\text { FPR, FNR, T= NA }\end{array}$ \\
\hline$[114]$ & 2018 & $\begin{array}{l}\text { Convolutional denoising } \\
\text { autoencoder networks }\end{array}$ & Multi-type & Multi-type defects & $\begin{array}{l}\text { labeling large } \\
\text { amounts of defective } \\
\text { samples are usually } \\
\text { harsh and } \\
\text { impracticable. }\end{array}$ & $\begin{array}{l}\text { Database } \\
\text { (PUB) }\end{array}$ & $\begin{array}{l}\text { Recall = } 0.6437 \\
\text { TPR }=0.638 \\
\text { F-Measure }=0.6279 \\
\text { FPR, FNR, T= NA }\end{array}$ \\
\hline$[115]$ & 2018 & $\begin{array}{l}\text { A Generic } \\
\text { Deep-Learning-Based } \\
\text { Approach }\end{array}$ & Hot-rolled strip & Multi-type defects & $\begin{array}{l}\text { collecting training } \\
\text { dataset is usually } \\
\text { costly }\end{array}$ & $\begin{array}{l}\text { Database } \\
\text { (PUB) }\end{array}$ & $\begin{array}{l}\mathrm{TPR}=0.992 \\
\mathrm{EER}=0.00 \\
\mathrm{FPR}, \mathrm{FNR}, \mathrm{T}=\mathrm{NA}\end{array}$ \\
\hline \multicolumn{4}{|c|}{$\begin{array}{l}\text { Notes: } \\
\text { Image source. } \\
\text { PUB: Public, PRI: private }\end{array}$} & \multicolumn{4}{|c|}{$\begin{array}{l}\text { Performance criteria. TPR: True positive rate, FPR: False positive rate, FNR: } \\
\text { False negative rate, EER: Equal error rate, AUC: Area under curve, MAE: } \\
\text { Mean absolute error, IoU: Intersection over union, MAP: Mean average } \\
\text { precision, T: Detection time }\end{array}$} \\
\hline
\end{tabular}

TABLE VIII

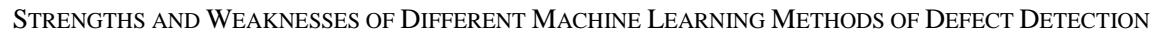

\begin{tabular}{|c|c|c|c|}
\hline Taxonomy & Methods & Strengths & Weaknesses \\
\hline \multirow{3}{*}{ Machine Learning } & Supervised learning & Has a good and reliable effect. & $\begin{array}{l}\text { Dependent on labeled samples, but defective } \\
\text { samples of flat steel are limited. }\end{array}$ \\
\hline & Unsupervised learning & Require no labeled samples for training. & $\begin{array}{l}\text { Susceptible to noise and highly influenced by } \\
\text { initial values. }\end{array}$ \\
\hline & Reinforcement learning & $\begin{array}{l}\text { Require only a small number of labeled samples and the } \\
\text { result is stable. }\end{array}$ & $\begin{array}{l}\text { Training requires a lot of interaction and reduces } \\
\text { efficiency. }\end{array}$ \\
\hline
\end{tabular}


the training images consist of a set of input vectors without any corresponding target values. Here, the unsupervised learning is dedicated to discover groups of similar examples within the input data. In some cases, it is also called clustering.

CNN can be used not only for supervised learning, but also for unsupervised learning. The deep convolutional generative adversarial network (DCGAN) [111] is a kind of CNN, which build certain constraints on traditional generative adversarial networks (GANs) to overcome its drawback of unstable output, and it often works in unsupervised learning manner for defect detection [112, 113]. Notably, Zhao et al. [113] combined GAN and autoencoder (AE) and LBP to detect defects on texture surface, which needs only positive samples without any defect sample nor manual label. This framework is of better practical application value due to its unsupervised natures. Moreover, AE-based algorithms also demonstrate strong competitiveness in steel surface defect detection, which are reported to be fairly noise-robust. Mei et al. [114] utilized convolutional denoising AE network to reconstruct image patches, combined with the reconstruction residual maps, this scheme can reliably learning final detection results, where no manual intervention is needed throughout all the detection process. Youkachen et al. [18] inventively applied convolutional auto-encoder (CAE) to reconstructed the defective test images, then the reconstructed images were used to highlight the shape feature by simple post-processing algorithms, providing another good application case on miscellaneous defect detection through unsupervised learning. Although the above unsupervised learning methods are able to learn from unlabeled images, they are susceptible to noise and initial value. How to consolidate the abovementioned impressive results into reliable achievements will become the focus of this branch direction.

\section{3) Reinforcement Learning}

Both supervised learning and unsupervised learning methods have obtained a rapid progress on surface defect detection of industrial flat steel. Different from these two methods, the reinforcement learning methods realize surface defect detection with fairly small datasets through a so-called rewards and punishment system to optimize inner parameters automatically. For example, Ren et al. [115] proposed a general approach requiring small training data for automated surface inspection. Authors transferred the features from a pretrained deep learning network and convolved the trained classifier over the input images. In the defect detection tests of flat steel surface, the proposed algorithm reduced error escape rates by from $6.00 \%$ to $19.00 \%$ in three defect types than several state-of-the-art benchmarks. Tao et al. [116] proposed a novel cascaded AE (CASAE) framework to detect some complex defects under industrial environment, which converts test images into pixel-wise prediction mask based on semantic segmentation. The defect regions can be accurately tracked by using a compact CNN. Zhou et al. [117] designed a new bilinear model of double-visual geometry group 16 (D-VGG16) to extract global and local features of surface defects, these features were then fed to the gradient-weighted class activation mapping (Grad-CAM) to finish defect detection. The proposed method can simultaneously realize defect classification and localization with small samples in weakly-supervised manner. Moreover, He et al. [118] proposed a new method named CAE-SGAN by fusing CAE and semi-supervised GAN (SGAN), where CAE acts as an advanced classifier to identify detective regions. The generalization ability improved by semi-supervised learning from SGAN supported that the CAE-SGAN scheme yielded competitive performance compared with some other traditional detection methods.

\section{4) Brief Summary}

Supervised learning determines test samples defective or non-defective by training samples with labels. Unsupervised learning can realize accurate and effective surface defect detection through the training of a large number of unlabeled samples in many harsh industrial manufacturing scenarios. In contrast, reinforcement learning tries to obtain intelligent self-optimization through continuously interacting with its environment, so as to achieve defect detection by making full use of limited labeled and unlabeled samples with low cost. For ease read, Table VII lists some typical defect detection methods based on machine learning with a short summary closely presented in Table VIII. As is stated above, machine learning tends to accomplish the defect detection tasks more intelligently, such an emerging technique is promising in the application of flat steel surface defect detection.

\section{SUMMARY AND DISCUSSION}

In Table I, III, V and VII, some typical defect detection methods among the four big families are highlighted. Attention is drawn to application scenarios, types of defects, involved challenges, source of images under test, reported detection performance. In terms of detection performance, on the one hand, detection accuracy is an important evaluation criterion. While, different references have different standard of detection accuracy, such as true positive rate (TPR), false negative rate (FNR), false positive rate (FPR), equal error rate (EER), area under curve (AUC), mean absolute error (MAE), G-mean, F-measure and so on. On the other hand, running time is another vital evaluation criterion, as the rapid casting or rolling rhythm of flat steel in real-world industrial sites has high-level time cost requirement on defect detection.

Respect to the image source used for study, the Raw images represent the real-world images (always with large size e.g., $4096 \times 1024$ pixel), which are acquired by an AVI machine running on industrial steel production line for defect detection. While the Database includes a number of defective or defect-free image block samples (always with small size, e.g., $256 \times 256$ pixel), which are obtained from Raw images after some post processes of segmentation and labeling. It is worth mentioning that the results of detection accuracy evaluated based on Raw images are more reliable than those evaluated based on Database, when the corresponding detection methods are really applied on AVI system in actual steel manufacturing line. And those will be more credible to flat steel manufacturers, as all the results of detection accuracy based on Raw images should be evaluated from all the detected defects and actual 
defects (not on Database, but on the real-world steel surface), while the actual defects need professional defect inspectors to find out one by one from the historical flat steel products, which are extremely labor-intensive and time-consuming. That is why this kind of studies (e.g. [5]) is quite rare at present. Driven by developments of emerging machine learning and improvements of hardware computing power, algorithmic research will develop towards the urgent needs of engineering applications, and more high-quality achievements can be expected to open in the near future.

Here in, this paper has summarized the research efforts made over the past two decades about the automated visual defect detection of flat steel surface in industrial manufacturing, where the largest volume of published reports in this literature belongs to the last five years. The research trend has gradually shifted from previous theoretical study to on-site application. Representative works from statistical, spectral, model-based and machine learning aspects are listed for readers to have a general overview of the state-of-the-arts. Existing challenges to surface defect detection and some potential proposals are investigated from a systematic perspective as follows.

1) How to make better balance of detection accuracy and computing efficiency is still relatively open to the automated computer-vision-based surface defect detection. But for the real-world industrial manufacturing of flat steels, detection stability especially robustness to environmental variations is on the very top list.

2) Real-time operation of high-resolution AVI system is expecting fast defect detection. As for algorithm itself, fusing features extracted by multiple descriptors to support final detection decision can yield better results than those produced by a single descriptor in the most cases. Intrinsic priors of production line are suggested to assist the defect detection. Online surface defect detection prefers lightweight arithmetic methods to complex learning networks, as our problem is an unsupervised and real-time detection task in essence. While machine learning or deep network is preeminent alternative for complex multi-class classification problem with rich datasets (i.e., defect classification). As the defect detection task can be treated as a bi-classification problem, it is not surprising that the machine learning trend is gradually sinking to the discussed defect detection topic. With respect to its resident hardware, the concept of edge computing could be employed for terminal accelerating, that is, ASICs such as FPGAs are encouraged to be placed at the front end of image acquisition where pre-processing on raw data can be finished in real time, so as to prevent redundant information being spread to the subsequent transmission and post-processing.

3) As the prelude of defect detection, noise smoothing and edge enhancing are suggested to be arranged as closer as possible to the imaging sensors, incredibly, the most effective denoising method for AVI system is to make the images as clean as possible by some feasible engineering measures. For example, equipping high pressure air-gun removing surface water droplets is far more effective than to develop advanced water removal algorithms to eliminate false alarm triggered by pseudo-defect. Moreover, adaptive and closed-loop controlling is strongly recommended for the illumination subsystem.

4) It is not prudent to compare detection performance of different techniques as different experiments select different testing methods with different evaluation criteria on distinct datasets. More steel surface defect databases, especially raw images from real-world industrial production line, are urgently expected for enriching diversified and cumulative future research ecology, which will be sure to benefit to explore for a feasible and comparable standard of performance evaluation for distinct defect detection methodologies.

We have tried to include as many as possible up-to-date references following the emerging AVI techniques, it is impossible to comprise all the existing publications due to space limitations. In addition, the second survey paper focusing on surface defect classification techniques about flat steels is under way.

\section{REFERENCES}

[1] S. Ghorai, A. Mukherjee, M. Gangadaran, and P. K. Dutta, "Automatic defect detection on hot-rolled flat steel products," IEEE Trans. Instrum. Meas., vol. 62, no. 3, pp. 612-621, Mar. 2013.

[2] K. Song, and Y. Yan, "A noise robust method based on completed local binary patterns for hot-rolled steel strip surface defects," Appl. Surf. Sci., vol. 285, pp. 858-864, Sep. 2013.

[3] Q. Luo, Y. Sun, P. Li, O. Simpson, L. Tian, and Y. He, "Generalized completed local binary patterns for time-efficient steel surface defect classification," IEEE Trans. Instrum. Meas., vol. 68, no. 3, pp. 667-679, Mar. 2019.

[4] D. He, K. Xu, P. Zhou, and D. Zhou, "Surface defect classification of steels with a new semi-supervised learning method," Opt. Lasers Eng., vol. 117, pp. 40-48, Feb. 2019.

[5] Q. Luo, and Y. He, "A cost-effective and automatic surface defect inspection system for hot-rolled flat steel," Robot. Comput. Integr. Manuf., vol. 38, pp. 16-30, Apr. 2016.

[6] R. Usamentiaga, D. F. Garcia, J. Molleda, F. G. Bulnes, and G. Bonet Brana, "Vibrations in steel strips: effects on flatness measurement and filtering," IEEE Trans. Ind. Appl., vol. 50, no. 5, pp. 3103-3112, Sep. 2014.

[7] G. Wu, H. Zhang, X. Sun, J. Xu, and K. Xu, "A Bran-new feature extraction method and its application to surface defect recognition of hot rolled strips," in IEEE Int. Conf. Autom. Logis., Jinan, China, Aug. 2007, pp. 2069-2074.

[8] A. Kumar, "Computer-vision-based fabric defect detection: a survey," IEEE Trans. Ind. Electron., vol. 55, no. 1, pp. 348-363, Jan. 2008.

[9] N. Neogi, D. K. Mohanta, and P. K. Dutta, "Review of vision-based steel surface inspection systems," EURASIP J. Image Video Process., vol. 2014, no. 1, pp. 50, Nov. 2014.

[10] C. A. Mack, "Fifty years of Moore's law," IEEE Trans. Semicond. Manuf., vol. 24, no. 2, pp. 202-207, Jun. 2011.

[11] R. T. Chin, and C. A. Harlow, "Automated visual inspection: a survey," IEEE Trans. Pattern Anal. Mach. Intell., vol. 4, no. 6, pp. 557-573, Dec. 1982.

[12] T. S. Newman, and A. K. Jain, "A survey of automated visual inspection," Comput. Vis. Image Underst., vol. 61, no. 2, pp. 231-262, Mar. 1995.

[13] X. Xie, "A review of recent advances in surface defect detection using texture analysis techniques," Elcvia Electron. Lett. on CV \& IA, vol. 7, no. 3, pp. 1-22, Apr. 2008.

[14] S. H. Huang, and Y. C. Pan, "Automated visual inspection in the semiconductor industry: A survey," Comput. Ind., vol. 66, pp. 1-10, Jan. 2015.

[15] W. B. Li, C. H. Lu, and J. C. Zhang, "A local annular contrast based real-time inspection algorithm for steel bar surface defects," Appl. Surf. Sci., vol. 258, no. 16, pp. 6080-6086, Mar. 2012.

[16] D. Fofi, F. Timm, P. R. Bingham, and E. Barth, "Non-parametric texture defect detection using Weibull features," in Proc. SPIE., San Francisco, CA, Feb. 2011. 
[17] H. Wang, J. Zhang, Y. Tian, H. Chen, H. Sun, and K. Liu, "A simple guidance template-based defect detection method for strip steel surfaces," IEEE Trans. Ind. Inform., vol. 15, no. 5, pp. 2798-2809, May 2019.

[18] S. Youkachen, M. Ruchanurucks, T. Phatrapomnant, and H. Kaneko, "Defect segmentation of hot-rolled steel strip surface by using convolutional auto-encoder and conventional image processing," in 10th IC-ICTES Bangkok, Thailand, Mar. 2019.

[19] G. Wu, H. Kwak, S. Jang, X. Ke, and J. Xu, "Design of online surface inspection system of hot rolled strips," in IEEE Int. Conf. Autom. Logis., Qingdao, China Sep. 2008, pp. 2291-2295.

[20] M. Sharifzadeh, S. Alirezaee, R. Amirfattahi, and S. Sadri, "Detection of steel defect using the image processing algorithms," in IEEE Int. Mult. Conf., Bahria Univ, Karachi, PAKISTAN, Feb. 2009, pp. 125-127.

[21] D. Djukic, and S. Spuzic, "Statistical discriminator of surface defects on hot rolled steel," Image Vis. Comput., pp. 158-163, Dec. 2007.

[22] G. K. Nand, Noopur, and N. Neogi, "Defect detection of steel surface using entropy segmentation," in 2014 Annual IEEE India Conf., Pune, India, Dec. 2015.

[23] N. Neogi, D. K. Mohanta, and P. K. Dutta, "Defect detection of steel surfaces with golbal adaptive percentile thresholding of gradient image," J. Ins. Eng., vol. 98, no. 6, pp. 557-565, Dec. 2017.

[24] F. G. Bulnes, R. Usamentiaga, D. F. Garcia, and J. Molleda, "Vision-based sensor for early detection of periodical defects in web materials," Sensors, vol. 12, no. 8, pp. 10788-809, Dec. 2012.

[25] Y. Zhao, Y. Yan, and K. Song, "Vision-based automatic detection of steel surface defects in the cold rolling process: considering the influence of industrial liquids and surface textures," Int. J. Adv. Manuf. Technol., vol. 90, no. 5-8, pp. 1665-1678, May 2016.

[26] J. Wang, Q. Li, J. Gan, H. Yu, and X. Yang, "Surface defect detection via entity sparsity pursuit with intrinsic priors," IEEE Trans. Ind. Inform., pp. 1-1, May 2019.

[27] A. Borselli, V. Colla, M. Vannucci, and M. Veroli, "A fuzzy inference system applied to defect detection in flat steel production," in IEEE Int. Conf. Fuz. Syst., Barcelona, Spain, Jan. 2010.

[28] T. Shi, J. Kong, X. Wang, Z. Liu, and G. Zheng, "Improved sobel algorithm for defect detection of rail surfaces with enhanced efficiency and accuracy," J. Cent. South Univ., vol. 23, no. 11, pp. 2867-2875, Nov. 2016.

[29] T. Bo, J. Kong, X. Wang, and C. Li, "Surface inspection system of steel strip based on machine vision," in 1st Int. Works. DBTA, Wuhan, Hubei, China, Apr. 2009, pp. 359-362.

[30] A. L. Zhiznyakov, D. G. Privezentsev, and A. A. Zakharov, "Using fractal features of digital images for the detection of surface defects," Pattern Recog. Image Anal., vol. 25, no. 1, pp. 122-131, Sep. 2015.

[31] M. Yazdchi, M. Yazdi, and A. G. Mahyari, "Steel Surface Defect Detection Using Texture Segmentation Based on Multifractal Dimension," in 2009 Int. Conf. DIP, Bangkok, Thailand, Mar. 2009, pp. 346-350.

[32] S. S. Yang, Y. H. He, Z. L. Wang, and W. S. Zhao, "A method of steel strip image segmentation based on local gray information," in IEEE Int. Conf. Ind. Tech., Chengdu, China, Apr. 2008.

[33] J. Choi, and C. Kim, "Unsupervised detection of surface defects: a two-step approach," in IEEE Int. Conf. Image Process., Orlando, FL, USA, Oct. 2012, pp. 1037-1040.

[34] Y. Ma, Q. Li, Y. Zhou, F. He, and S. Xi, "A surface defects inspection method based on multidirectional gray-level fluctuation," Int. J. Adv. Robot. Syst., vol. 14, no. 3, May 2017.

[35] R. Haralick, "Textural features for image classification," IEEE Trans. Syst. Man Cybern. Syst., vol. SMC-3, no. 6, pp. 610-621, Nov. 1973.

[36] J. J. Zhou, R. Y. Guo, M. T. Sun, T. Di, S. Wang, J. Y. Zhai, and Z. Zhao, "The effects of GLCM parameters on LAI estimation using texture values from quickbird satellite imagery," Sci. Rep., vol. 7, Aug. 2017.

[37] Z. K. Xing, and H. M. Jia, "Multilevel color image segmentation based on GLCM and improved salp swarm algorithm," IEEE Access, vol. 7, pp. 37672-37690, 2019.

[38] R. B. Vallabhaneni, and V. Rajesh, "Brain tumour detection using mean shift clustering and GLCM features with edge adaptive total variation denoising technique," Alex. Eng. J., vol. 57, no. 4, pp. 2387-2392, Dec. 2018.

[39] Y. Wang, H. Xia, X. Yuan, L. Ling, and S. Bei, "Distributed defect recognition on steel surfaces using an improved random forest algorithm with optimal multi-feature-set fusion," Multimed. Tools Appl., vol. 77, no. 13, pp. 16741-16770, Jul. 2017.
[40] D.-M. Tsai, M.-C. Chen, W.-C. Li, and W.-Y. Chiu, "A fast regularity measure for surface defect detection," Mach. Vis. Appl., vol. 23, no. 5, pp. 869-886, Sep. 2012

[41] M. Chu, and R. Gong, "Invariant feature extraction method based on smoothed local binary pattern for strip steel surface defect," ISIJ Int., vol. 55, no. 9, pp. 1956-1962, May 2015.

[42] T. Ojala, M. Pietikainen, and D. Harwood, "A comparative study of texture measures with classification based on feature distributions," Pattern Recognit., vol. 29, no. 1, pp. 51-59, Jan. 1996.

[43] M. Chu, R. Gong, S. Gao, and J. Zhao, "Steel surface defects recognition based on multi-type statistical features and enhanced twin support vector machine," Chemometr. Intell. Lab. , vol. 171, pp. 140-150, Dec. 2017.

[44] Y. Liu, K. Xu, and D. Wang, "Online surface defect identification of cold rolled strips based on local binary pattern and extreme learning machine," Metals, vol. 8, no. 3, Mar 2018

[45] J. Zhao, Y. Peng, and Y. Yan, "Steel surface defect classification based on discriminant manifold regularized local descriptor," IEEE Access, vol. 6, pp. 71719-71731, Nov. 2018.

[46] Q. Luo, X. Fang, Y. Sun, L. Liu, J. Ai, C. Yang, and O. Simpson, "Surface defect classification for hot-rolled steel strips by selectively dominant local binary patterns," IEEE Access, vol. 7, pp. 23488-23499, Feb. 2019.

[47] F. Dupont, C. Odet, and M. Cartont, "Optimization of the recognition of defects in flat steel products with the cost matrices theory," Ndt \& E Int., vol. 30, no. 1, pp. 3-10, Feb. 1997.

[48] M. González-Hidalgo, S. Massanet, A. Mir, and D. Ruiz-Aguilera, "Improving salt and pepper noise removal using a fuzzy mathematical morphology-based filter," Appl. Soft. Comput., vol. 63, pp. 167-180, Feb. 2017

[49] H. Zheng, L. X. Kong, and S. Nahavandi, "Automatic inspection of metallic surface defects using genetic algorithms," J. Mater. Process. Tech., vol. 125, no. 36, pp. 427-433, Sep. 2002.

[50] C. Doo-Chul, J. Yong-Ju, Y. Jong Pil, and K. S. Woo, "Pinhole detection in steel slab images using Gabor filter and morphological features," Appl. Optics, vol. 50, no. 26, pp. 5122-5129, Sep. 2011.

[51] X. Li, S. K. Tso, X. Guan, and Q. Huang, "Improving automatic detection of defects in castings by applying wavelet technique," IEEE Trans. Ind. Electron., vol. 53, no. 6, pp. 1927-1934, Dec. 2006.

[52] M. Liu, Y. Liu, H. Hu, and L. Nie, "Genetic algorithm and mathematical morphology based binarization method for strip steel defect image with non-uniform illumination," J. Vis. Commun. Image Represent., vol. 37, pp. 70-77, May 2016.

[53] K. Song, S. Hu, Y. Yan, and J. Li, "Surface defect detection method using saliency linear scanning morphology for silicon steel strip under oil pollution interference," ISIJ Int., vol. 54, no. 11, pp. 2598-2607, Jul. 2014

[54] D. Chetverikov, and A. Hanbury, "Finding defects in texture using regularity and local orientation," Pattern. Recogn., vol. 35, no. 10, pp. 2165-2180, 2002.

[55] B. R. Suresh, R. A. Fundakowski, T. S. Levitt, and J. E. Overland, "A real-time automated visual inspection system for hot steel slabs," IEEE Trans. Pattern Anal. Mach. Intell., vol. 5, no. 6, pp. 563-572, Dec. 1983.

[56] Y. h. Ai, and K. Xu, "Surface detection of continuous casting slabs based on curvelet transform and kernel locality preserving projections," J. Iron Steel Res. Int., vol. 20, no. 5, pp. 80-86, May 2013.

[57] "The Phase Only Transform for unsupervised surface defect detection," in 2010 IEEE conf. comput. vis. pattern. recong. , San Francisco, 2010, pp. 295-302.

[58] X. Shufu, S. Shiguang, C. Xilin, and C. Jie, "Fusing local patterns of Gabor magnitude and phase for face recognition," IEEE Trans. Image Process., vol. 19, no. 5, pp. 1349-1361, Jun. 2010.

[59] J. Kim, S. Um, and D. Min, "Fast 2D complex Gabor filter with kernel decomposition," IEEE Trans. Image Process., vol. 27, no. 4, pp. 1713-1722, Apr. 2018.

[60] J. Borg, S. Gershon, and M. Alpert, "Optimal Gabor filters for textile flaw detection," Pattern Recognit., vol. 35, no. 12, pp. 2973-2991, Dec. 2002.

[61] Z. Ni, H. Zeng, L. Ma, J. Hou, J. Chen, and K. Ma, "A Gabor feature-based quality assessment model for the screen content images," IEEE Trans. Image Process., vol. 27, no. 9, pp. 4516-4528, Sep. 2018.

[62] Y. Jeon, D. Choi, J. Yun, and S. Kim, "Detection of periodic defects using dual-light switching lighting method on the surface of thick plates," ISIJ Int., vol. 55, no. 9, pp. 1942-1949, May 2015.

[63] J. P. Yun, D. Kim, K. Kim, S. J. Lee, C. H. Park, and S. W. Kim, "Vision-based surface defect inspection for thick steel plates," Opt. Eng., vol. 56, no. 5, May 2017. 
[64] D. c. Choi, Y. J. Jeon, S. H. Kim, S. Moon, J. P. Yun, and S. W. Kim, "Detection of pinholes in steel slabs using Gabor filter combination and morphological features," ISIJ Int., vol. 57, no. 6, pp. 1045-1053, May 2017.

[65] R. Medina, F. Gayubo, L. M. González-Rodrigo, D. Olmedo, J. Gómez-García-Bermejo, E. Zalama, and J. R. Perán, "Automated visual classification of frequent defects in flat steel coils," Int. J. Adv. Manuf. Technol., vol. 57, no. 9-12, pp. 1087-1097, Dec. 2011.

[66] A. S. Tolba, "Fast defect detection in homogeneous flat surface products," Expert Syst. Appl., vol. 38, no. 10, pp. 12339-12347, Sep. 2011

[67] G. Alvaro, B. Joao, and M. Odemir, "Gabor wavelets combined with volumetric fractal dimension applied to texture analysis," Pattern. Recogn. Lett., vol. 36, pp. 135-143, Oct. 2014.

[68] T. Randen, and J. H. Hus, "Texture segmentation using filters with optimized energy separation," IEEE Trans. Image Process., vol. 8, no. 4, pp. 571-582, Apr. 2002.

[69] A. Kumar, "Automated defect defection in textured materials," Ph.D. dissertation, Dept. Elect. Electron. Eng., Univ.Hong Kong, Hong Kong, China, May 2001.

[70] A. Kumar, and G. K. H. Pang, "Defect detection in textured materials using optimized filters," IEEE Trans. Syst. Man, Cybern. B, Cybern., vol. 32, no. 5, pp. 553-70, Oct. 2002.

[71] A. Kumar, "Inspection of surface defects using optimal FIR filters," in Proc. ICASSP, Hong Kong, China, Apr. 2003, pp. 241-244.

[72] Y. J. Jeon, D. C. Choi, S. J. Lee, J. P. Yun, and S. W. Kim, "Steel-surface defect detection using a switching-lighting scheme," Appl. Optics, vol. 55 , no. 1, pp. 47-57, Jan. 2016

[73] W. Liu, and Y. Yan, "Automated surface defect detection for cold-rolled steel strip based on wavelet anisotropic diffusion method," Int. J. Ind. Syst. Eng., vol. 17, no. 2, pp. 224-239, Feb. 2014.

[74] X. Wu, K. Xu, and J. Xu, "Application of undecimated wavelet transform to surface defect detection of hot rolled steel plates," in Int. Congress Image Signal Process., Sanya, China, May. 2008, pp. 528-532.

[75] K. Song, S. Hu, and Y. Yan, "Automatic recognition of surface defects on hot-rolled steel strip using scattering convolution network," J. Comput. Inf. Syst., vol. 10, no. 7, pp. 3049-3055, Apr. 2014.

[76] L. P. Erwan, and M. Stéphane, "Sparse geometric image representations with bandelets," IEEE Trans. Image Process., vol. 14, no. 4, pp. 423-438, Apr. 2005.

[77] J. Krommweh, "Tetrolet transform: a new adaptive Haar wavelet algorithm for sparse image representation," J. Vis. Commun. Image Represent., vol. 21, no. 4, pp. 364-374, May 2010.

[78] X. Zhang, Y. H. Yan, W. H. Chen, and J. J. Chen, "Image fusion method for strip steel surface detect based on Bandelet-PCNN," Adv. Mater. Res., pp. 806-810, Jul. 2012.

[79] K. Xu, S. Liu, and Y. Ai, "Application of Shearlet transform to classification of surface defects for metals," Image Vis. Comput., vol. 35, pp. 23-30, Mar 2015.

[80] K. Xu, Y. Xu, P. Zhou, and L. Wang, "Application of RNAMlet to surface defect identification of steels," Opt. Lasers Eng., vol. 105, pp. 110-117, Jun. 2018.

[81] E. J. Candes, Donoho, L. David, and advisor, "Ridgelets : theory and applications," Icase/larc, 1998.

[82] E. Candès, L. Demanet, D. Donoho, and L. Ying, "Fast discrete Curvelet transforms," Multiscale Model. Sim., vol. 5, no. 3, pp. 861--899, Sep. 2006.

[83] R. C. Gonzalez, and R. E. Woods, Digital Image Processing (3rd Edition), 2007

[84] X. Si, J. Feng, J. Zhou, and Y. Luo, "Detection and rectification of distorted fingerprints," IEEE Trans. Pattern Anal. Mach. Intell., vol. 37, no. 3, pp. 555-68, Mar. 2015.

[85] S. Gu, J. Feng, J. Lu, and J. Zhou, "Efficient Rectification of Distorted Fingerprints," IEEE Trans. Inf. Forensic Secur., vol. 13, no. 1, pp. 156-169, Jan. 2018.

[86] T. D. DuaJl, D. A. Duc, and T. L. H. Du, "Combining hough transform and contour algorithm for detecting vehicles' license-plates," in Proc. 2004 Int. Sym. Intell. Mult. Video, Hong Kong, China,, Jun. 2005.

[87] G. R. Cross, and A. K. Jain, "Markov random field texture models," IEEE Trans. Pattern Anal. Mach. Intell., vol. 5, no. 1, pp. 25-29, Feb. 1983.

[88] F. Gayubo, J. L. González, E. De La Fuente, F. Miguel, and J. R. Perán, "On-line machine vision system for detect split defects in sheet-metal forming processes," in Int. Conf. Pattern Recognit, Hong Kong, China, Aug. 2006, pp. 723-726.
[89] K. Xu, "Application of hidden Markov tree model to on-line detection of surface defects for steel strips," J. Mech. Eng., vol. 49, no. 22, pp. 34-40, Nov. 2013.

[90] I. Ben Ayed, N. Hennane, and A. Mitiche, "Unsupervised variational image segmentation/classification using a Weibull observation model," IEEE Trans. Image Process., vol. 15, no. 11, pp. 3431-3439, Nov. 2006.

[91] K. Liu, H. Wang, H. Chen, E. Qu, Y. Tian, and H. Sun, "Steel surface defect detection using a new Haar-Weibull-variance model in unsupervised manner," IEEE Trans. Instrum. Meas., vol. 66, no. 10, pp. 2585-2596, Jul. 2017.

[92] B. Wu, and Y. Yang, "Local- and global-statistics-based active Contour model for Image segmentation," Math. Probl. Eng., vol. 2012, pp. 1-16, Apr. 2012.

[93] L. Wang, Y. Chang, H. Wang, Z. Wu, J. Pu, and X. Yang, "An active contour model based on local fitted images for image segmentation," Inf. Sci., vol. 418-419, pp. 61-73, Dec. 2017.

[94] K. Song, and Y. Yan, "Micro surface defect detection method for silicon steel strip based on saliency convex active Contour model," Math. Probl. Eng., vol. 2013, pp. 1-13, Nov. 2013.

[95] J. Yang, X. Li, J. Xu, Y. Cao, Y. Zhang, L. Wang, and S. Jiang, "Development of an optical defect inspection algorithm based on an active contour model for large steel roller surfaces," Appl. Optics, vol. 57, no. 10, pp. 2490-2498, Apr. 2018.

[96] S. Susan, and M. Sharma, "Automatic texture defect detection using Gaussian mixture entropy modeling," Neurocomputing, vol. 239, pp. 232-237, May. 2017.

[97] H. Yan, K. Paynabar, and J. Shi, "Anomaly Detection in Images With Smooth Background via Smooth-Sparse Decomposition," Technometrics, vol. 59, no. 1, pp. 102-114, 2017.

[98] Q. Huangpeng, H. Zhang, X. Zeng, and W. Huang, "Automatic visual defect detection using texture prior and low-rank representation," IEEE Access, vol. 6, pp. 37965-37976, Jul. 2018.

[99] S. Zhou, S. Wu, H. Liu, Y. Lu, and N. Hu, "Double low-rank and sparse decomposition for surface defect segmentation of steel sheet," Appl. Sci., vol. 8, no. 9, pp. 1628-1643, Sep. 2018.

[100]D. C. Choi, Y. J. Jeon, S. J. Lee, J. P. Yun, and S. W. Kim, “Algorithm for detecting seam cracks in steel plates using a Gabor filter combination method," Appl Opt, vol. 53, no. 22, pp. 4865-72, Aug. 2014.

[101]M. W. Ashour, F. Khalid, A. Abdul Halin, L. N. Abdullah, and S. H. Darwish, "Surface defects classification of hot-rolled steel strips using multi-directional Shearlet features," Arab. J. Sci. Eng., vol. 44, no. 4, pp. 2925-2932, 2018.

[102]H. B. Liu, and G. W. Kang, "Surface defects inspection of cold rolled strips based on neural network," in Int. Conf. Mach. Learn. Cybern., Canton, China, Aug. 2005, pp. 5034-5037.

[103]W. Daniel, S. Bernd, and S. Moshe, "Design of deep convolutional neural network architectures for automated feature extraction in industrial inspection," Cirp Annals-Manuf. Technol, vol. 65, no. 1, pp. 417-420, 2016.

[104]J. Park, B. Kwon, J. Park, and D. Kang, "Machine learning-based imaging system for surface defect inspection," Inter. J. of Prec. Eng. Manuf-Green Technol., vol. 3, no. 3, pp. 303-310, 2016.

[105]Y. Cha, and W. Choi, "Deep learning-based crack damage detection using convolutional neural networks," Comput.-Aided. Civil. Inf. Eng., vol. 32, no. 5, pp. 361-378, Sep. 2017.

[106]D. He, K. Xu, and P. Zhou, "Defect detection of hot rolled steels with a new object detection framework called classification priority network," Comput. Ind. Eng., vol. 128, pp. 290-297, Dec. 2018.

[107]Y. Liu, K. Xu, and J. Xu, "Periodic surface defect detection in steel plates based on deep learning," Appl. Sci., vol. 9, no. 15, Aug. 2019.

[108]L. Song, W. Lin, Y. Yang, X. Zhu, Q. Guo, and J. Xi, "Weak micro-scratch detection based on deep convolutional neural network," IEEE Access., vol. 7, pp. 27547-27554, Feb. 2019.

[109]Y. Cha, W. Choi, G. Suh, and S. Mahmoudkhani, "Autonomous structural visual inspection using region-based deep learning for detecting multiple damage types," Comput.-Aided. Civil. Inf. Eng., vol. 33, no. 9, pp. 731-747, Nov. 2017.

[110]J. Li, Z. Su, J. Geng, and Y. Yin, "Real-time detection of steel strip surface defects based on improved YOLO detection network," in IFAC Works. MMM, Shanghai, 2018, pp. 76-81.

[111]A. Radford, and L. Metz, "Unsupervised representation learning with deep convolutional generative adversarial networks," in Comput. Sci., 2015 . 
[112]T. Schlegl, P. Seeböck, S. Waldstein, U. Schmidt-Erfurth, and G. Langs, "Unsupervised anomaly detection with generative adversarial networks to guide marker discovery," in IPMI 2017: Inf. Process. Med. Imag., 2017, pp. 146-157.

[113]Z. Zhao, B. Li, R. Dong, and P. Zhao, "A surface defect detection method based on positive samples," PRICAI 2018: Trends. AI, Lecture Notes in Computer Science, pp. 473-481, 2018.

[114]S. Mei, H. Yang, and Z. Yin, "An unsupervised-learning-based approach for automated defect inspection on textured surfaces," IEEE Trans. Instrum. Meas., vol. 67, no. 6, pp. 1266-1277, Jun. 2018.

[115] R. Ren, T. Hung, and K. Tan, "A generic deep-learning-based approach for automated surface inspection," IEEE Trans. Cybern., vol. 48, no. 3, pp. 929-940, Mar. 2018.

[116]X. Tao, D. Zhang, W. Ma, X. Liu, and D. Xu, "Automatic metallic surface defect detection and recognition with convolutional neural networks," Appl. Sci., vol. 8, no. 9, Sep. 2018.

[117]F. Zhou, G. Liu, F. Xu, and H. Deng, "A generic automated surface defect detection based on a bilinear model," Appl. Sci., vol. 9, no. 15, pp. 3159-3175, Aug. 2019.

[118]D. He, K. Xu, P. Zhou, and D. Zhou, "Surface defect classification of steels with a new semi-supervised learning method," Opt. Las. Eng., vol. 117, pp. 40-48, Feb. 2019.

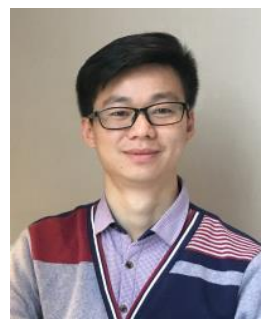

QIWU LUO (M'17) received the B.S. degree in communication engineering from the National University of Defense Technology, Changsha, China, in 2008, and the M.Sc. degree in electronic science and technology and the Ph.D. degree in electrical engineering from Hunan University, Changsha, in 2011 and 2016, respectively.

He was a Senior Engineer of instrumentation with WASION Group Ltd. Company, Changsha, China, and the Deputy Technical Director with Hunan RAMON Technology Co., Ltd., Changsha. In 2016, he joined the School of Electrical Engineering and Automation, Hefei University of Technology, Hefei, China, where he also completed his postdoctoral research on automatic optic inspection (AOI). He is currently an Associate Professor with the School of Automation, Central South University, Changsha, China. His current research interests include computer vision, industrial AOI, machine learning, parallel hardware architecture design and reconfigurable computing.

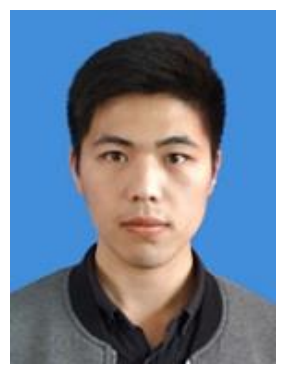

XIAOXIN FANG received the B.S. degree in electrical engineering and automation from the Jiangsu University of Science and Technology in 2018, He is currently pursuing the M.Sc. degree under the guidance of Dr. Luo with the School of Electrical Engineering and Automation, Hefei University of Technology, Hefei, China.

His current research interests include texture analysis and image classification.

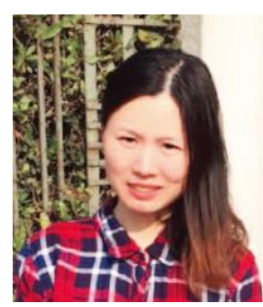

LI LIU received the B.Sc. degree in communication engineering, the M.Sc. degree in photogrammetry and remote sensing and the Ph.D. degree in information and communication engineering from the National University of Defense Technology (NUDT), China, in 2003, 2005 and 2012, respectively.

She joined the faculty at NUDT in 2012, where she was an Associate Professor with the College of System Engineering. Currently she is working at the University of Finland. During her Ph.D. study, she spent more than two years as a Visiting Student at the University of Waterloo, Canada, from 2008 to 2010. From 2015 to 2016, she spent ten months visiting the Multimedia Laboratory at the Chinese University of Hong Kong. From Dec. 2016 to Sep. 2018, she worked as a senior researcher at the Machine Vision Group at the University of Oulu, Finland.

Dr. Liu was a cochair of International Workshops at ACCV2014, CVPR2016, ICCV2017, ECCV2018 and CVPR2019. She is organizing a tutorial on Textures, Objects and Scenes at CVPR 2019. She was a guest editor of special issues for IEEE TPAMI and IJCV. Her current research interests include facial behavior analysis, texture analysis, image classification, object detection and recognition. Her papers have currently over 1800 citations in Google Scholar. She currently serves as Associate Editor of the Visual Computer Journal.

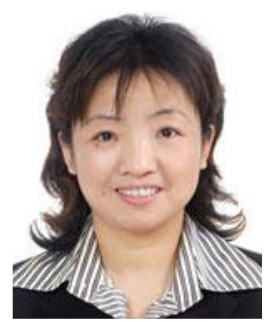

CHUNHUA YANG (M'09) received the M.S. degree in automatic control engineering and the Ph.D. degree in control science and engineering from Central South University, Changsha, China, in 1988 and 2002, respectively.

From 1999 to 2001, she was a Visiting Professor with the University of Leuven, Leuven, Belgium. Since 1999, she has been a Full Professor with the School of Information Science and Engineering, Central South University, Changsha, China. From 2009 to 2010, she was a Senior Visiting Scholar with the University of Western Ontario, London, ON, Canada. She is currently the HoD of the School of Automation, Central South University. Her current research interests include modeling and optimal control of complex industrial processes, and intelligent control systems.

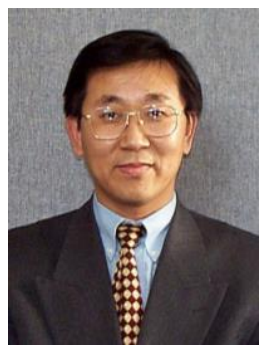

YICHUANG SUN (M'90-SM'99) received the B.Sc. and M.Sc. degrees from Dalian Maritime University, Dalian, China, in 1982 and 1985, respectively, and the Ph.D. degree from the University of York, York, U.K., in 1996, all in communications and electronics engineering.

He is currently a Professor of Communications and Electronics, the Head of Communications and Intelligent Systems Research Group, and the Head of Electronic, Communication and Electrical Engineering Division in the School of Engineering and Computer Science of the University of Hertfordshire, UK. He has published over 330 papers and contributed 10 chapters in edited books. He has also published four text and research books: Continuous-Time Active Filter Design (CRC Press, USA, 1999), Design of High Frequency Integrated Analogue Filters (IEE Press, UK, 2002), Wireless Communication Circuits and Systems (IET Press, 2004), and Test and Diagnosis of Analogue, Mixed-signal and RF Integrated Circuits - the Systems on Chip Approach (IET Press, 2008). His research interests are in the areas of wireless and mobile communications, $\mathrm{RF}$ and analogue circuits, microelectronic devices and systems, and machine learning and deep learning.

Prof. Sun was a Series Editor of IEE Circuits, Devices and Systems Book Series (2003-2008). He has been Associate Editor of IEEE Transactions on Circuits and Systems I: Regular Papers (2010-2011, 2016-2017, 2018-2019). $\mathrm{He}$ is also Editor of ETRI Journal, Journal of Semiconductors, and Journal of Sensor and Actuator Networks. He was Guest Editor of eight IEEE and IEE/IET journal special issues: High-frequency Integrated Analogue Filters in IEE Proc. Circuits, Devices and Systems (2000), RF Circuits and Systems for Wireless Communications in IEE Proc. Circuits, Devices and Systems (2002), Analogue and Mixed-Signal Test for Systems on Chip in IEE Proc. Circuits, Devices and Systems (2004), MIMO Wireless and Mobile Communications in IEE Proc. Communications (2006), Advanced Signal Processing for Wireless and Mobile Communications in IET Signal Processing (2009), Cooperative Wireless and Mobile Communications in IET Communications (2013), Software-Defined Radio Transceivers and Circuits for 5G Wireless Communications in IEEE Transactions on Circuits and Systems-II (2016), and the 2016 IEEE International Symposium on Circuits and Systems in IEEE Transactions on Circuits and Systems-I (2016). He has also been widely involved in various IEEE technical committee and international conference activities. 